\title{
On the relevance of subcritical hydrodynamic turbulence to accretion disk transport
}

\author{
G. Lesur and P.-Y. Longaretti
}

Laboratoire d'Astrophysique, Observatoire de Grenoble, BP 53, 38041 Grenoble Cedex 9, France

e-mail: [geoffroy.lesur; pierre-yves.longaretti]@obs.ujf-grenoble.fr

Received 22 June 2005 / Accepted 13 September 2005

\begin{abstract}
Hydrodynamic unstratified Keplerian flows are known to be linearly stable at all Reynolds numbers, but may nevertheless become turbulent through nonlinear mechanisms. However, in the last ten years, conflicting points of view have appeared on this issue. We have revisited the problem through numerical simulations in the shearing sheet limit. It turns out that the effect of the Coriolis force in stabilizing the flow depends on whether the flow is cyclonic (cooperating shear and rotation vorticities) or anticyclonic (competing shear and rotation vorticities); Keplerian flows are anticyclonic. We have obtained the following results:

i/ The Coriolis force does not quench turbulence in subcritical flows; however, turbulence is more efficient, and much more easily found, in cyclonic flows than in anticyclonic ones.

ii/ The Reynolds number/rotation/resolution relation has been quantified in this problem. In particular we find that the resolution demand, when moving away from the marginal stability boundary, is much more severe for anticyclonic flows than for cyclonic ones. Presently available computer resources do not allow numerical codes to reach the Keplerian regime.

iii/ The efficiency of turbulent transport is directly correlated to the Reynolds number of transition to turbulence $R g$, in such a way that the Shakura-Sunyaev parameter $\alpha \sim 1 / R g$. This correlation is nearly independent of the flow cyclonicity. The correlation is expected on the basis of generic physical arguments.

iv/ Even the most optimistic extrapolations of our numerical data show that subcritical turbulent transport would be too inefficient in Keplerian flows by several orders of magnitude for astrophysical purposes. Vertical boundary conditions may play a role in this issue although no significant effect was found in our preliminary tests.

v/ Our results suggest that the data obtained for Keplerian-like flows in a Taylor-Couette settings are largely affected by secondary flows, such as Ekman circulation.
\end{abstract}

Key words. accretion, accretion disks - hydrodynamics - instabilities - turbulence

\section{Introduction}

The question of the existence and physical origin of turbulence in accretion disks has been lively debated for a number of decades. Generally speaking, there are a priori two basic ways in which an accretion disk can become turbulent. In the first way, some linear instability is present in the flow, and its nonlinear development eventually drives turbulence. In the second one, the flow is linearly stable, and undergoes a direct laminarturbulent transition once a certain threshold in Reynolds number is reached. The first type of transition to turbulence is called supercritical, and the second, (globally) subcritical.

Global instabilities (such as the Papaloizou \& Pringle 1984 instability) seem unpromising to drive turbulence (Blaes 1987; Hawley 1991). As for local instabilities, an astrophysically important example of supercritical transition is provided by the magneto-rotational instability (MRI) which has been extensively studied following the pioneering work of Balbus, Hawley and their collaborators (Balbus \& Hawley 1991; Hawley et al. 1995; see Balbus 2003, for a recent review). The turbulent transport induced by this instability is by now characterized in a number of instances, and has been called upon even when only some fraction of the disk is ionized, as in the midplane region of YSOs inner disks - the dead-zone (Gammie 1996; Fleming \& Stone 2003). However, the reduced efficiency of the transport in this case, as well as the possible existence of disks which may not support MHD phenomena at all, has prompted some upsurge of interest in purely hydrodynamic instabilities. A local, baroclinic-like instability has been observed in global simulations by Klahr \& Bodenheimer (2003). Local stability analyzes (Klahr 2004; Johnson \& Gammie 2005a) find transient instability in this context, but shearing box simulations indicate that this does not drive turbulence (Johnson \& Gammie 2005b). Urpin (2003) discusses an instability related 
to vertical shear and heat transport of the Goldreich-Schubert type (Goldreich \& Schubert 1967); however, this instability produces only a rather weak radial transport (Arlt \& Urpin 2004). More recently, Dubrulle et al. (2005b) and Shalybkov $\&$ Ruediger (2005) have discussed an instability arising when both the fluid differential rotation and vertical stratification are stabilizing according to the Høiland criterion. However, it seems that this instability is connected to the presence of walls, and is dynamically important only when the inter-wall distance is small enough for a resonant-like interaction to take place $^{1}$ (Satomura 1981), otherwise disturbances are confined to the near boundary zone; a related result has recently been found in the astrophysics literature (Umurhan 2005). Earlier analytic and numerical investigations have shown this instability to be absent in local disk models (Goodman \& Balbus 2001; Brandenburg \& Dintrans 2001; Rüdiger et al. 2002). Note finally that vertical convection in a stratified disk can in principle also drive turbulence; however, it induces inwards transport instead of the required outwards one (Cabot 1996; Stone \& Balbus 1996). Therefore, no local instability has yet been found in the hydrodynamic regime, which would explain the turbulent transport taking place in accretion disks.

Subcritical transition to turbulence is the subject of the present work. The non-rotating plane Couette flow provides a classical (and to date the best understood) example of a system undergoing a subcritical transition. Although the nature and mechanism of the transition remained elusive for decades, it has been identified in the recent years, in laboratory experiments (Daviaud et al. 1992; Dauchot \& Daviaud 1995a,b; Bottin et al. 1997), numerical simulations (Hamilton et al. 1995; see also Schmiegel \& Eckhardt 1997 and Eckhardt \& Mersmann 1999), and theoretical analyzes (in particular Waleffe 1997; Waleffe 2003). Earlier investigations of the problem have focused on the role of nonlinear instabilities in subcritical shear flows, based on Landau-like toy-models on the one hand (e.g., Drazin \& Reid 1981 and references therein), and analysis of the linear stability of finite amplitude defects in the flow profile on the other (Lerner \& Knobloch 1988; Dubrulle \& Zahn 1991; Dubrulle 1993); unfortunately, such analyzes yield little information on the existence and location of the turbulent state in parameter space and on the turbulent transport efficiency, unless further ad hoc assumptions are made.

In any case, on the basis of the empirically observed subcritical transition in laboratory flows, it was suggested that a similar process is relevant in accretion disks (Shakura et al. 1978), in spite of their very different prevailing physical conditions. This suggestion was tested and challenged in a series of numerical simulations performed by Balbus et al. (1996) and Hawley et al. (1999), in the shearing sheet limit. Transition to turbulence was not found in these simulations for Keplerianlike flows. The simulations were performed with two different finite difference codes (a PPM type code, and the ZEUS code), up to a resolution of $256^{3}$. These two works concluded that a stabilizing Coriolis force prevents the existence of turbulence

\footnotetext{
${ }^{1}$ We thank Stéphane Le Dizes for bringing this point to our attention.
}

in the simulated flows, except in the immediate vicinity of the linear marginal stability limits.

This conclusion was in turn questioned by Richard \& Zahn (1999), on the basis of the Taylor-Couette experiments performed by Wendt (1933) and Taylor (1936). These experimental results display a subcritical transition to turbulence in presence of a stabilizing Coriolis force. Also, new sets of experiments have been carried out in order to bring the experimental conditions closer to the ones prevailing in a Keplerian flow. Namely, a Taylor-Couette apparatus was used in conditions of radially decreasing angular velocity and radially increasing specific angular momentum. Turbulence was again found for high enough Reynolds numbers (Richard 2001; Richard et al. 2001) but the results are not unambiguous, as the potential role of secondary flows induced by the boundary conditions in the experiments, such as Ekmann's circulation, is unclear, in spite of the attention devoted to this point in the experiments. In any case, a subcritical transition is also found in all experiments of shear flows on which a linearly stabilizing Coriolis force is superimposed (Longaretti \& Dauchot 2005).

Longaretti (2002) has argued from a phenomenological analysis that the lack of turbulence in the simulations performed to date was due to a lack of resolution, as the Coriolis force may increase the range of scales that need to be resolved for a subcritical turbulent transition to show up. On the other hand, on the basis of a newly developed Reynolds stress closure scheme (Ogilvie 2003), Garaud \& Ogilvie (2005) find that Keplerian flows may or may not be turbulent depending on the parameters of the scheme. For their favored choice of parameters, unbounded Keplerian flows are not turbulent, on the contrary to linearly stable, wall-bounded Taylor-Couette flows.

The recent astrophysical literature on the problem of subcritical transition has also focused on the concept of transient growth in Keplerian flows (Chagelishvili et al. 2003; Tevzadze et al. 2003; Yecko 2004; Umurhan \& Regev 2004; Mukhopadhyay et al. 2005; Afshordi et al. 2005). Due to the nonnormal character of the Navier-Stokes equation, linear modes can transiently be strongly amplified in shear flows, although on the long run they must viscously decay. It has been argued that this transient growth can be relevant to astrophysical disks in two different ways. First, 3D turbulence (or an external forcing) can couple to large scale 2D structures; the (statistical) amplitude of these structures can be large, under the combined action of this coupling, of transient growth and of viscous decay, and these 2D structures may contribute to the overall transport in the disk (Ioannou \& Kakouris 2001). Secondly, a large transient growth has been invoked in the bypass scenario of transition to turbulence, which involves an interplay between nonnormality and nonlinearity (see, e.g., Grossman 2000; Brosa \& Grossmann 1999). Waleffe (1995) has emphasized the key role played by nonlinear interactions in the context of the recently identified turbulent self-sustaining process of non-rotating plane Couette flows (Hamilton et al. 1995; Waleffe 1997). Even though transient growth explains the strong modulations of the streamwise velocity from relatively weak streamwise rolls involved in this self-sustaining mechanism, the existence and properties of the turbulent basin of attraction for the full nonlinear dynamics are 
apparently poorly constrained by the nonnormal linear problem characteristics.

Our present understanding of the possible existence of a dynamically significant subcritical turbulent transition in accretion disks is unsatisfying in several respects, calling for a reinvestigation of the problem. On the one hand, the relevance of the available laboratory experiments to accretion disk turbulence is at best unclear, as will be shown in the course of the present work (for a different opinion, see Hersant et al. 2005). On the other hand, the absence of subcritical turbulence in the shearing sheet local model of accretion disks used by Balbus et al. (1996) and Hawley et al. (1999) may be an effect of various numerical limitations, namely, algorithm choice, limited resolution, nature of the boundary conditions, imposed aspect ratio and initial conditions of the simulations. Of these options, only the first two have been partially addressed in these previous investigations, leading to questions concerning the "effective Reynolds number" of the performed simulations - an ill-defined process-dependent concept, that we shall clarify in the context of the present problem. Following the suggestion of Longaretti (2002), the primary aim of the present work is to investigate in a more systematic way, through numerical simulations of plane parallel, rotating shear flows, the effects of finite resolution on the results. The effects of the other factors listed above are also somewhat explored, but to a lesser extent. Both cyclonic and anticyclonic rotation are considered; although cyclonic rotation is not relevant to accretion disks, it turns out that cyclonic flows behave very differently from anticyclonic ones, opening some interesting perspective into the nature of the problem.

This paper is organized as follows. Section 2.1 collects the background material relevant to the problem. First, the form of the equations solved is provided, and the global energy budget recalled, before discussing linear stability limits. The section is concluded by a summary of the effect of a stabilizing rotation in shear flows as characterized by the available laboratory experiments. The next section presents the various codes used in this work, and the numerical results obtained with them. Section 4 discusses various aspects of our numerical results, most notably the role of resolution and boundary conditions on the numerical side, the role of the Coriolis force, the underlying phenomenological picture, and the astrophysical implications, on the physical side. A summary is provided in Sect. 5, along with an outlook on the question of turbulence in accretion disks.

\section{Rotating plane shear flows: a summary}

The present investigation is concerned with the nonlinear instability of laminar flows characterized by a uniform shear, in the presence of a uniform global rotation. The direction of the flow is identified with the $x$ axis (streamwise direction), and the direction of the shear with the $y$ axis (shearwise direction); rotation is applied along the $z$ axis (spanwise direction). The laminar flow $\boldsymbol{u}_{L}$ is invariant in the streamwise and spanwise directions (in particular, the vertical stratification expected in a real disk is ignored): $\boldsymbol{u}_{L}=U(y) \boldsymbol{e}_{x}$.

Such a flow can be used to numerically model either a local portion of an accretion disk, or experiments on rotating plane
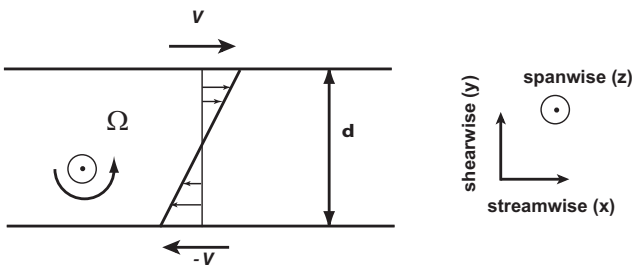

Fig. 1. Sketch of the configuration of rotating plane shear flows.

Couette flows, depending on the nature of the applied boundary condition in the shearwise direction (in practice, either rigid or shearing sheet; see next section). The configuration is represented in Fig. 1.

\subsection{Equations of motion}

The most useful form of the Navier-Stokes equation, for our present purpose, is obtained by separating the laminar flow $\boldsymbol{u}_{L}$ and the deviation from laminar $\boldsymbol{w}$ in the total velocity $\boldsymbol{u}$ in the rotating frame, leading to

$$
\begin{aligned}
\frac{\partial \boldsymbol{w}}{\partial t}+\boldsymbol{w} \cdot \boldsymbol{\nabla} w=S \cdot y \frac{\partial \boldsymbol{w}}{\partial x} & +(2 \Omega+S) w_{y} \boldsymbol{e}_{x}-2 \Omega w_{x} \boldsymbol{e}_{y} \\
& -\frac{\boldsymbol{\nabla} \delta \pi}{\rho}+v \Delta \boldsymbol{w}
\end{aligned}
$$

where the gradient terms balancing the laminar flow Coriolis force has been subtracted out to form the effective generalized pressure $\delta \pi$ (which therefore absorbs the equilibrium centrifugal, gravitational and/or pressure force term, depending on the considered equilibrium problem); $\Omega$ is the flow rotation velocity in an inertial frame, and $S=-\mathrm{d} U / \mathrm{d} y$ is the shear. The convention adopted here is that the sign of $S$ is chosen to be positive when the flow is cyclonic, i.e., when the contributions of shear and rotation to the flow vorticity have the same sign. With our choice of axes, this implies that $S=-2 S_{x y}$, where $S_{i j}=1 / 2\left(\partial_{i} u_{L, j}+\partial_{j} u_{L, i}\right)$ is the usual deformation tensor. The system is closed either with the usual continuity equation supplemented by a polytropic equation of state, or, for simplicity, through an incompressibility assumption $(\boldsymbol{\nabla} \cdot \boldsymbol{w}=0)$.

The relevant global time-scales of the problem are the shear time-scale $t_{\mathrm{s}}=\left|S^{-1}\right|$, the viscous one $t_{v}=d^{2} / v$ ( $d$ is the gap in the experiment, or the shearwise size of the shearing sheet box), and the rotation time-scale related to the Coriolis force $t_{\Omega}=(2 \Omega)^{-1}$; they relate to the advection term, the viscous term, and the Coriolis force term, respectively. Correlatively, the flow is described by two dimensionless numbers, the Reynolds number

$\operatorname{Re}=t_{v} / t_{\mathrm{s}}=|S| d^{2} / v$,

and the rotation number

$R_{\Omega}=\operatorname{sgn}(S) t_{\mathrm{s}} / t_{\Omega}=2 \Omega / S$.

For Keplerian flows, $R_{\Omega}=-4 / 3$. More generally, if one assumes that the large scale rotation of an astrophysical disk follows a power-law, $\Omega(r) \propto r^{-q}$, one locally has $R_{\Omega}=-2 / q$ in the disk. 
Note that our Reynolds number is defined on the outer scales, and not on the turbulent ones, such as, e.g., the Taylor microscale. Large values $\left(\sim 10^{4}\right)$ of this number are involved in the problem investigated here; the correlative numerical requirements are discussed in Sect. 4.4.

\subsection{Energy budget}

As the global energy budget plays some role in the discussion of the results, it is rederived here. In the following equations, the bracket notation refers to a volume average of the bracketed quantity. The averaging volume is the simulation one, and shearing-sheet boundary conditions are assumed in the derivation, for definiteness. For the kinetic energy in the streamwise and shearwise directions, one finds:

$$
\begin{aligned}
\frac{\partial}{\partial t}\left\langle\frac{w_{x}^{2}}{2}\right\rangle= & S\left(R_{\Omega}+1\right)\left\langle w_{x} w_{y}\right\rangle \\
& -\left\langle\frac{w_{y}}{\rho} \frac{\partial \delta \pi}{\partial x}\right\rangle+v\left\langle w_{x} \Delta w_{x}\right\rangle,
\end{aligned}
$$

$$
\begin{aligned}
\frac{\partial}{\partial t}\left\langle\frac{w_{y}^{2}}{2}\right\rangle= & -S R_{\Omega}\left\langle w_{x} w_{y}\right\rangle \\
& -\left\langle\frac{w_{y}}{\rho} \frac{\partial \delta \pi}{\partial y}\right\rangle+\nu\left\langle w_{y} \Delta w_{y}\right\rangle .
\end{aligned}
$$

Instead of the vertical equation, it is more instructive to write down the total kinetic energy equation:

$\frac{\partial}{\partial t}\left\langle\frac{\boldsymbol{w}^{2}}{2}\right\rangle=S\left\langle w_{x} w_{y}\right\rangle-\epsilon$

where

$$
\epsilon=v \sum_{i}\left\langle\left(\nabla w_{i}\right)^{2}\right\rangle
$$

is the usual energy injection rate of turbulence cascade arguments $^{2}$. In this last equation the incompressibility condition and the boundary conditions have been used in the reexpression of the pressure term, and an integration by part has been performed on the viscous term (a constant kinematic viscosity $v$ is assumed).

In statistical steady-state, Eq. (6) reduces to,

$S\left\langle w_{x} w_{y}\right\rangle=\epsilon$.

As pointed out by Balbus et al. (1996), the fact that $\epsilon>0$ implies that in steady state, the shear rate and the Reynolds stress responsible for radial transport have identical signs. This result has a direct physical interpretation: the imposed shear prevents the flow to be in global thermodynamic equilibrium. Nevertheless, the flow tries to restore this global equilibrium by radially transporting momentum through the turbulent Reynolds stress from regions of larger momentum to regions of lower momentum, consistently with Eq. (8).

\footnotetext{
${ }^{2}$ Because the rate of energy transfer in scale is constant in a Kolmogorov-like argument, the injection rate is directly related to the small-scale dissipation rate.
}

Note finally that, in Eqs. (4) and (5), the pressure-velocity correlation terms cannot be neglected, as they are of the order of the cascade energy injection term $\epsilon$. This is almost unavoidable, as pressure is the only force that can provide for the acceleration of fluid particles in turbulent motions. As a matter of fact, the energy budget of any particular velocity component depends critically on the behavior of the velocity-pressure correlations, which are notoriously difficult to model (Speziale 1991). Ignoring this term in the analysis of the energetics therefore leads to dubious or erroneous conclusions.

\subsection{Linear stability limits}

Surprisingly enough, the question of the linear stability limits of the simple rotating shear flows considered here is not completely solved to date. Focusing for the time being on purely streamwise-independent perturbations, instability with respect to local perturbations follows when (Pedley 1969; Leblanc \& Cambon 1997; Sipp \& Jacquin 2000)

$R_{\Omega}\left(R_{\Omega}+1\right)<0$,

or, equivalently, $-1<R_{\Omega}<0$.

In plane Couette flows, it has been proven that $R_{\Omega}^{+} \equiv$ 0 is the correct cyclonic marginal stability limit for non streamwise-invariant perturbations as well, at all Reynolds numbers (Romanov 1973). No such generic proof exists at the anticyclonic marginal stability limit $\left(R_{\Omega}^{-} \equiv-1\right)$. However, various linear and nonlinear numerical investigations suggest that this is indeed the case (Cambon et al. 1994; Komminaho et al. 1996; Bech \& Andersson 1997). These results belong to plane Couette flows with rigid boundary conditions in the shearwise direction, but tend to prove that a local criterion captures the correct stability limit, as observed, e.g., in the simulations of Balbus et al. (1996) and Hawley et al. (1999).

The physics behind Eq. (9) can be captured by a displaced particle argument (Tritton \& Davies 1981; Tritton 1992). This argument is reproduced in Appendix A for the reader's convenience. Note that Eq. (9) is identical to Rayleigh's specific angular momentum criterion for the centrifugal instability, as the usual epicyclic frequency reads $\kappa^{2}=S^{2} R_{\Omega}\left(1+R_{\Omega}\right)$. However, in the plane shear flow limit of cylindrical flows, the concept of specific angular momentum used in the derivation of Rayleigh's criterion no longer has meaning, so that one must follow a different route, as done here. Note also that, consequently, the Rayleigh criterion for the centrifugal instability in the inertial frame can also be understood from the action of the Coriolis force in the rotating frame (a somewhat surprising, although not new conclusion), as the displaced particle argument of Appendix A is readily extended to cylindrical flows.

\subsection{Subcritical transition in rotating plane Couette flows: a summary of relevant experimental results}

In the laboratory, non-rotating plane Couette flows undergo a subcritical transition to turbulence at $\mathrm{Re} \simeq 1500$. The transition Reynolds number steeply increases if a stabilizing rotation and/or a curvature is superimposed on the flow. The conceptually cleanest way to add rotation to a plane Couette flow is 
to place a plane Couette apparatus on a rotating table. Also, by considering a Taylor-Couette apparatus with varying gap width and independently rotating cylinders, one obtains a flow in which both rotation and curvature effects can be studied, and which reduces to a rotating plane Couette flow in the narrow gap limit. For a more complete discussion of the distinction and characterization of rotation and curvature in Taylor-Couette experiments, and of the related experimental data, the reader is referred to Longaretti \& Dauchot (2005).

For the range of parameters studied to date in the experiments, it turns out that rotation and curvature effects on the transition Reynolds number are superposed in an mostly additive way, so that both plane Couette flows and Taylor-Couette flows can in principle be used to characterize the effect of rotation. Concerning cyclonic flows, the only directly relevant data have been collected by Tillmark \& Alfredsson (1996) with the help of a plane Couette flow apparatus placed on a rotating table. For anticyclonic flows, the only available experiments are those of Richard and coworkers (Richard 2001; Richard et al. 2001), who used a Taylor-Couette apparatus. The range of rotation number $R_{\Omega}$ explored in these experiments is 0 to 0.1 for cyclonic rotation, and -1.6 to -1 for anticyclonic rotation. The data are shown in Fig. 2

The important point to note here is the steep dependence of the transition Reynolds number with the "distance" to marginal stability, with a typical slope $\left|\Delta R_{g}\right| /\left|\Delta R_{\Omega}\right| \sim 10^{4}-10^{5}$.

\section{Numerical codes, strategy, and results}

In the present work, we are concerned with rotating, unstratified uniform shear flows. Periodic boundary conditions hold in the direction of the flow ( $x$ axis) and the "vertical" direction ( $z$ axis), and either shearing sheet or rigid boundary conditions are applied in the direction of the shear ( $y$ direction). The vertical axis is also the axis of rotation of the flow. The shearing sheet boundary conditions are described in detail by Hawley et al. (1995). Shearing sheet flows thus modelled can be viewed as a local approximation of disk flows, while the use of mixed rigid-periodic boundary conditions is appropriate to numerically represent the rotating plane Couette flows of laboratory experiments, as routinely done in the fluid mechanics community.

\subsection{Numerical codes}

Two different 3D codes have been written for the present work: a finite difference compressible code, similar to ZEUS (Stone \& Norman 1992), but restricted to the Cartesian geometry, and rigid-periodic or shearing sheet boundary conditions; and a 3D incompressible Fourier code, in Cartesian geometry, and implementing only the shearing sheet boundary conditions. An explicit kinematic viscosity term is added in both codes, upon which the Reynolds number is defined. Both codes were parallelized using the Message Passing Interface.

The shearing sheet boundary conditions induce some changes with respect to a standard Fourier code. As a matter of fact, while we were developing this code, the work by Umurhan \& Regev (2004) appeared, which implements the
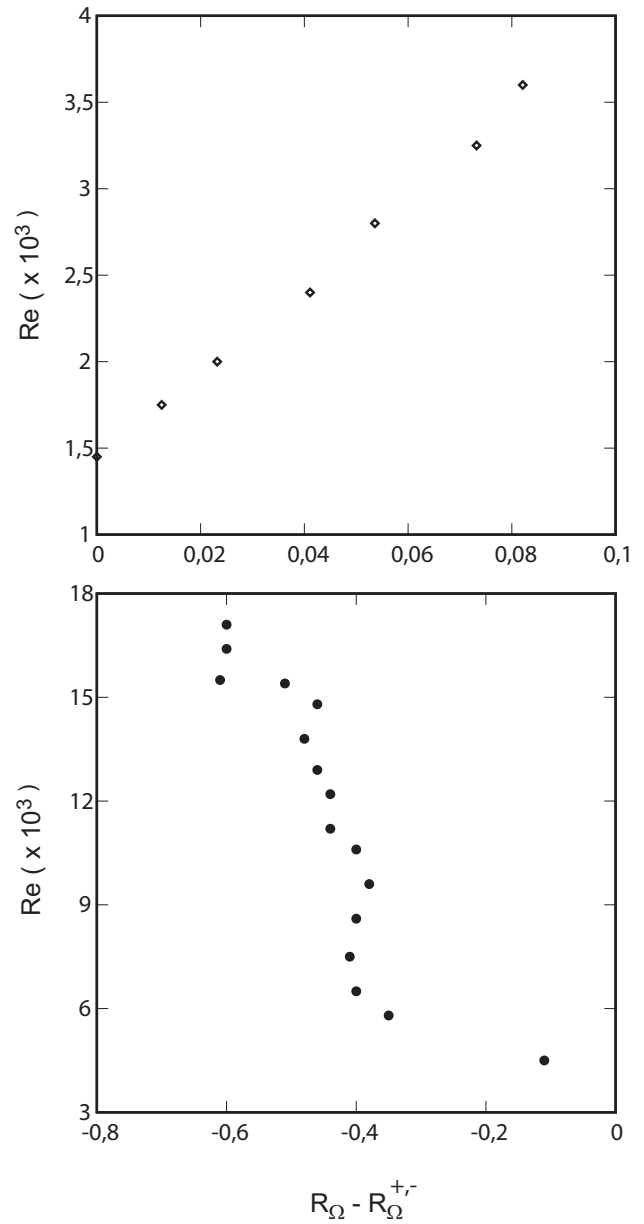

Fig. 2. Data on the Reynolds number of subcritical transition to turbulence as a function of the rotation number $R_{\Omega}$, measured from the appropriate marginal stability limit $R_{\Omega}^{ \pm}$(see text). Top panel: cyclonic plane Couette flow (data from Tillmark \& Alfredsson 1996). Bottom panel: anticyclonic Taylor-Couette flow (data from Richard 2001). The anticyclonic data are more difficult to collect, and consequently noisier.

same technique. Therefore, our description of the required changes will be brief, and we refer the reader to this recent paper for details.

To get effective periodic boundary conditions on the 3 axes, one needs to write Eq. (1) in the sheared frame defined by:

$t^{\prime}=t$

$x^{\prime}=x+S \cdot y \cdot t$

$y^{\prime}=y$

$z^{\prime}=z$.

In this shearing frame, Eq. (1) (supplemented by the incompressibility condition) becomes:

$$
\begin{aligned}
\frac{\partial \boldsymbol{w}}{\partial t^{\prime}}+\boldsymbol{w} \cdot \tilde{\nabla} \boldsymbol{w}= & -\frac{\tilde{\boldsymbol{\nabla}} \delta \pi}{\rho}-2 \Omega w_{x} \boldsymbol{e}_{\boldsymbol{y}}+(2 \Omega+S) w_{y} \boldsymbol{e}_{\boldsymbol{x}} \\
& +v \tilde{\Delta} \boldsymbol{w} \\
\tilde{\boldsymbol{\nabla}} \cdot \boldsymbol{w}= & 0
\end{aligned}
$$

in which $\tilde{\boldsymbol{\nabla}}=\partial_{x^{\prime}} \boldsymbol{e}_{x^{\prime}}+\left(\partial_{y^{\prime}}-S t^{\prime} \partial_{x^{\prime}}\right) \boldsymbol{e}_{y^{\prime}}+\partial_{z^{\prime}} \boldsymbol{e}_{z^{\prime}}$ and $\tilde{\Delta}=\tilde{\boldsymbol{\nabla}} \cdot \tilde{\boldsymbol{\nabla}}$. 
Since the shearing box is a periodic box in the shearing frame, this last formulation of the Navier-Stokes equation can be written in 3D-Fourier Space. Defining:

$\boldsymbol{\mu}=\boldsymbol{k}-S t k_{x} \boldsymbol{e}_{y}$,

one finally obtains:

$$
\begin{aligned}
\frac{\partial \hat{w}}{\partial t^{\prime}}+\mathrm{i} \boldsymbol{\mu} \cdot \widehat{\boldsymbol{w} \otimes \boldsymbol{w}}= & -\mathrm{i} \boldsymbol{\mu} \frac{\widehat{\delta \pi}}{\rho}-2 \Omega \hat{w}_{x} \boldsymbol{e}_{y}+(2 \Omega+S) \hat{w}_{y} \boldsymbol{e}_{\boldsymbol{x}} \\
& -v \mu^{2} \boldsymbol{w} \\
\boldsymbol{\mu} \cdot \hat{\boldsymbol{w}}= & 0 .
\end{aligned}
$$

These are the equations actually used in our spectral code. The nonlinear term is computed using the $2 / 3$ dealiasing rule with a pseudo-spectral method (see e.g. Peyret 2002 for a description of this point) and each time-step is evaluated using a 4th order Runge Kutta Scheme. One should note that a k-wave in the sheared frame actually appears as a $\mu(t)$-wave in the steady frame. Then, as time goes on in the simulation, the k-grid describes higher spatial frequency in the steady frame and consequently, the large scales are not computed anymore. Since nonlinear coupling limits the shearing of any wave-number, a remap procedure is periodically applied all along the simulation, and prevents to loose information on the large scale 3 (Rogallo 1981). This kind of algorithm has been extensively described by Umurhan \& Regev (2004) using a 2D spectral code and the reader should refer to this publication for technical details on the remap procedure.

The choice of these two codes was made first for purposes of comparison with previous work, and secondly to allow us to cross-check the potential limitations of one code against the other; e.g., the shearing sheet boundary conditions and sheared spatial basis Eq. (16) have their own limitations, as the sheared basis forms a complete basis for shearing sheet boundary conditions, but only for these conditions.

The three codes were tested in a variety of ways. The first test was to reproduce the non-rotating plane Couette flow behavior computed by Hamilton et al. (1995). This was done both with our finite difference code, and with David Clarke's version of ZEUS3D, for comparison purposes. We checked the nonlinear transition mechanism was well reproduced, with the corresponding Reynolds number and aspect ratio, and that the two codes gave completely consistent results. Then, the shearing sheet boundary conditions were tested using these two finite difference codes and the Fourier code. We have verified that mean turbulent quantities (e.g., mean energy, mean transport, velocity maxima and minima) and critical Reynolds number were statistically the same using the different codes, for different rotation numbers, either cyclonic or anticyclonic. This consistency holds over the $10^{5}-10^{6}$ time steps of our simulations.

\subsection{Initial conditions and numerical strategy}

The experimental results recalled in Sect. 2.4 suggest that a steep dependence of the transition Reynolds number with the rotation number may be the cause of the difficulty to find such

\footnotetext{
${ }^{3}$ We thank Achim Wirth for pointing out this reference to us.
}

a transition in the previously published shearing sheet numerical simulations. Accordingly, one of the major aims of this investigation is to quantify the effect of the simulation resolution on the determination of the transition Reynolds number as a function of $R_{\Omega}$.

Now, one of the characteristic features of the subcritical transition to turbulence is an observed spread in transition Reynolds numbers, depending on the choice of initial conditions, and a correlative large spread in turbulence life-times. This has been documented both experimentally (Darbyshire \& Mullin 1995) and numerically (Faisst \& Eckhardt 2004) in pipe flows, and guides to some extent our choice of initial conditions and our numerical procedure. Indeed, turbulent life-times typically vary from fast decay (survival for less than one hundred dynamical times) to long or indefinite survival (several thousands of dynamical times, with a clear divergence at finite Reynolds number) over several orders of magnitude of variation of the initial condition amplitude, but for less than 50\% of variations of Reynolds number (see Faisst \& Eckhardt 2004, Figs. 2 and 7).

It is reasonable to assume that this qualitative behavior is generic. Consequently, we have chosen once and for all, fixed, high amplitude initial conditions, to make our numerical runs more directly comparable to one another upon variations of Reynolds numbers. Furthermore, we consider that turbulence is long-lived if it is not observed to decay for 100 or 200 shear times (depending on the runs). This choice is a compromise between computational time constraints, and accuracy in the determination of the transition Reynolds number of indefinitely self-sustained turbulence. In practice, simulations are performed in a cubic box (the impact of this choice is discussed in the next section, to some extent). The flow is adimensionalized with the only dimensional quantities introduced in the problem: $S$ and $d$, where $d$ is the simulation box size (or equivalently, by choosing $|S|=1$ and $d=1$ ). The initial conditions used for all our simulation are a random 3D excitation of the 10 largest Fourier modes, with rms fluctuations in velocity of order unity in our chosen units. Other shapes of initial conditions were tested such as white noise (all scales excited randomly) or introducing large scale vortices in various directions with a small superimposed noise. This produces no significant difference once the flow is relaxed $\left(t \gtrsim 20 t_{\mathrm{s}}\right.$ ).

The numerical strategy adopted is then rather straightforward: choosing a code, a resolution, a boundary condition (for the finite difference code) and a Reynolds number, at fixed initial conditions, the flow evolution is computed starting from the marginal stability limit in rotation number $R_{\Omega}$ and evolving the rotation number by (small) fixed steps every 100 or 200 shear times. According to the preceding discussion, this allows us to reduce at maximum the number of runs and the run time needed to observe systematic trends in the numerical results.

In this section, only shearing sheet boundary conditions are used. We have also checked that the time required to dissipate the turbulent energy of the flow assuming energy injection is stopped (deduced from the $\epsilon$ term in Eq. (6)) is smaller than $100 t_{s}$; this constraint is always satisfied by a large margin in all our runs, implying that the deviations from laminar motion that we observe are self-sustained (i.e., we do not 

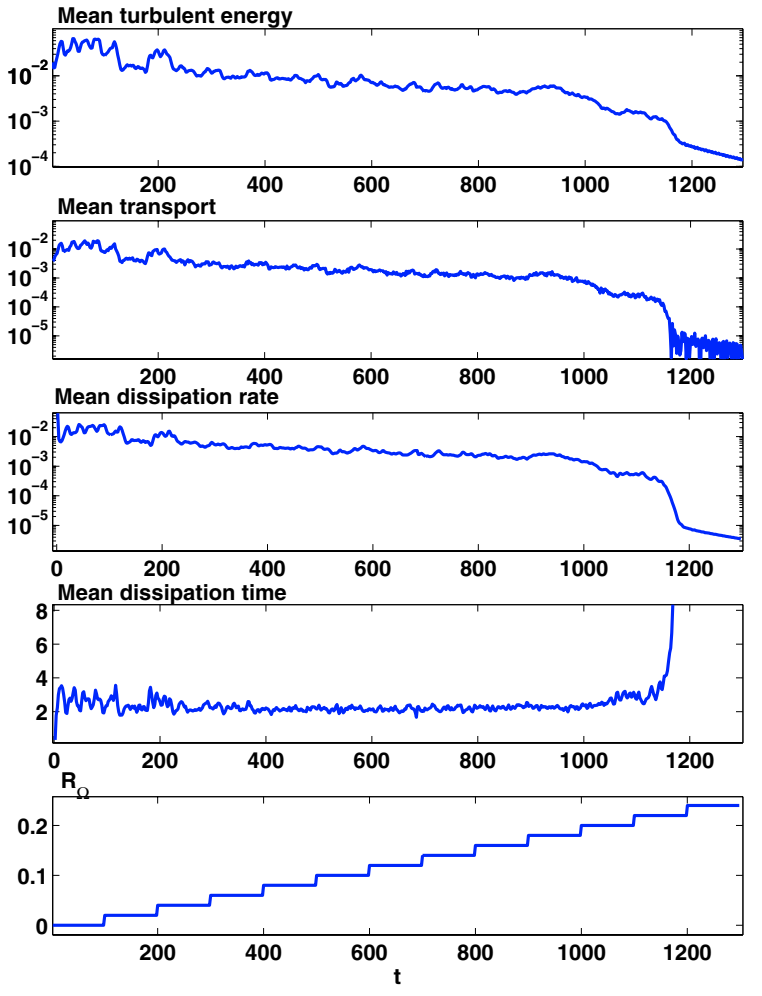

Fig. 3. Example of the time evolution of a $64^{3}(\mathrm{Re}=12000)$ cyclonic flow run as computed by our Fourier code. The turbulent energy, transport and dissipation rate are the quantities involved in Eq. (6). The dissipation time follows from the turbulent energy and the dissipation rate. The bottom panel displays the evolution of the rotation number that is imposed in the course of the simulation.

observe them because their dissipation time exceeds the run time). Actually, once turbulence is lost in our simulations, the energy in the velocity fluctuations always decreases rather fast, as can be checked in Fig. 3 for cyclonic flows. The same property is found for anticyclonic flows, see Sect. 3.4.

We conclude this section on our choice of the Mach number $\left(\mathrm{Ma}=d S / c_{\mathrm{s}}\right)$ for our simulations with the compressible Zeus-like code. The type of motions we are considering in these simulations reach at most a small fraction of the boundaries relative velocity (normalized to unity in this work). We found that a sound speed also normalized to unity was a good compromise between limiting the effects of compressibility (which eventually makes the turbulence compressible and largely different in character when the Mach number is too large), and the impact of the sound speed on the CFL condition. Also, this value mimics the real role of compressibility in a vertically stratified accretion disk. Consequently, $\mathrm{Ma}=1$ is imposed in all our compressible simulations.

\subsection{Numerical results: cyclonic flows}

On the cyclonic side, simulations are performed while maintaining the rotation number $R_{\Omega}$ constant during 100 shear times; then the rotation number is increased by steps of 0.02 , starting from the marginal cyclonic point $R_{\Omega}=0$. An example global output of such a simulation is plotted in Fig. 3

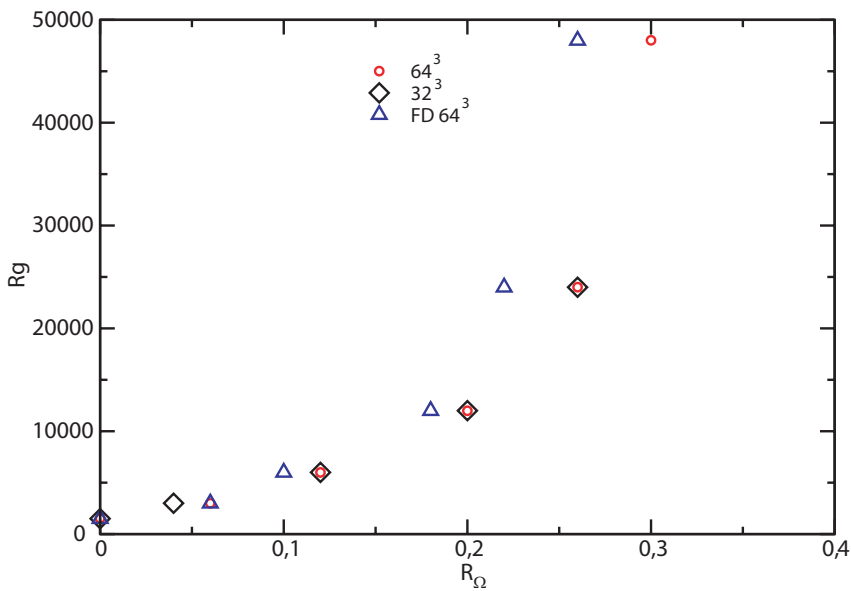

Fig. 4. Transition Reynolds number $R g$ as a function of the rotation number $R_{\Omega}$, with different resolutions and codes for shearing-sheet boundary conditions (cyclonic rotation). All points were obtained using our Fourier code except those labelled FD (finite difference) which use our ZEUS-like code.

for $\mathrm{Re}=12000$. The relaminarization point is easily found since the transition between the turbulent to laminar state is quite abrupt (at $t=1150$ in Fig. 3). We define the last turbulent point as the last rotation rate for which turbulence is sustained for $100 t_{\mathrm{s}}$. For our example simulation, we find that the last turbulent point at $\operatorname{Re}=12000$ and $64^{3}$ resolution with our Fourier code is $R_{\Omega}=0.2$.

Using this kind of simulation, we plot the last turbulent points in the $\left(\operatorname{Re}, R_{\Omega}\right)$ space, for different resolutions and/or codes in Fig. 4. Turbulence is found on the cyclonic side at least up to $R_{\Omega}=0.3$, i.e. significantly away from the marginal stability point.

Note that turbulence is maintained with certainty (with our adopted criteria) at any given point, but, due to the sampling made in the explored Reynolds number, turbulence may also be maintained at a somewhat lower Reynolds number (i.e. just below the last turbulent point in Fig. 3). This can be true down to the previously tested Reynolds number, for which turbulence is not maintained at the considered rotation rate. In conclusion, the real transition Reynolds $R g$ curve in the $\left(R_{\Omega}, \mathrm{Re}\right)$ plane should be found somewhat below (but not far from) the last turbulent point curve determined here. This remark is more important for anticyclonic flows, for which precise quantitative results are needed.

Except for a systematic shift between the results obtained with the Fourier code and the ZEUS-like one, the results seem to be independent of the resolution. The numerically minded reader may ask how one can reach such high Reynolds numbers with such relatively small resolutions. This point is addressed in Sect. 4.4.

An important issue is to quantify transport in subcritical turbulent flows. The phenomenological arguments of Longaretti (2002) suggest that $\left\langle v_{x} v_{y}\right\rangle \propto 1 / R g$ in subcritical flows, and that the turbulent transport in a given flow with specified $\left(\operatorname{Re}, R_{\Omega}\right)$ 


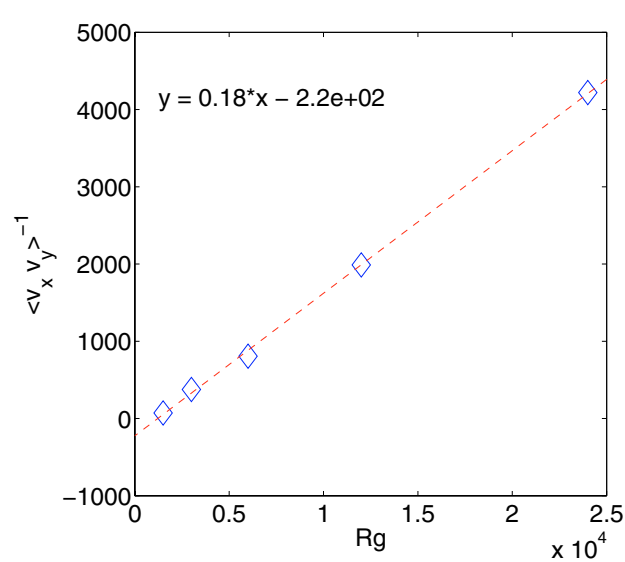

Fig. 5. Mean transport as a function of the transition Reynolds number for cyclonic rotation (normalized by $S^{2} d^{2}$ ).

numbers depends only on $R_{\Omega}$ through $R g$ (see Sect. 4.1$)^{4}$. Consequently, we have used all our simulations at a given $R_{\Omega}$ to obtain the least noisy evaluation of $\left\langle v_{x} v_{y}\right\rangle$. Then, with the help of Fig. 3, one finds a transition Reynolds number $R g$ for any given $R_{\Omega}$, which allows us to plot the mean turbulent transport $\left\langle v_{x} v_{y}\right\rangle$ as a function of the transition Reynolds number in Fig. 5. This was done only from the data of our Fourier code for self-consistency, but using both the $32^{3}$ and $64^{3}$ resolution runs, as they produced the same results, and as the use of a larger data set improves the statistics. The resulting relation reads

$\left\langle v_{x} v_{y}\right\rangle \simeq \frac{5.5}{R g-1250}(S d)^{2}$.

The presence of an additive constant in the denominator of this expression is a clear indication of the influence of the linear instability close to the marginal stability limit; indeed, transport in the supercritical region is significantly enhanced with respect to the subcritical region (see, e.g., Fig. 16 in Dubrulle et al. 2005a, and explanations therein). For large critical Reynolds number (i.e., far enough from the marginal stability boundary, e.g., $R g \gtrsim 15000),\left\langle v_{x} v_{y}\right\rangle \simeq 5.5 / R g$ is a good approximation.

\subsection{Numerical results: anticyclonic flows}

The strategy adopted in simulations of anticyclonic flows is similar to the cyclonic side. Starting at $R_{\Omega}=-1.0$, the rotation number is decreased in steps of 0.004 and each step lasts 200 shear times to allow for flow relaxation. A typical run is shown in Fig. 6, computed with our 3D Fourier Code at $\operatorname{Re}=12000$. One should note that the flow fluctuations have higher amplitudes on the anticyclonic side than on the cyclonic side; this is why we have reduced the rotation number steps and increased the relaxation time in anticyclonic runs. Consistently, The last turbulent point is defined here as the last rotation rate for which turbulence is sustained for $200 t_{\mathrm{s}}$. On the

\footnotetext{
4 The same result follows if one assumes that in the fully turbulent state, the torque $\propto \mathrm{Re}^{2}$, as predicted in Kolmogorov turbulence, and observed in experiments (see, e.g., Dubrulle et al. 2005a). The argument of Sect. 4.1 allows us to recover this result from more generic physical principles.
}

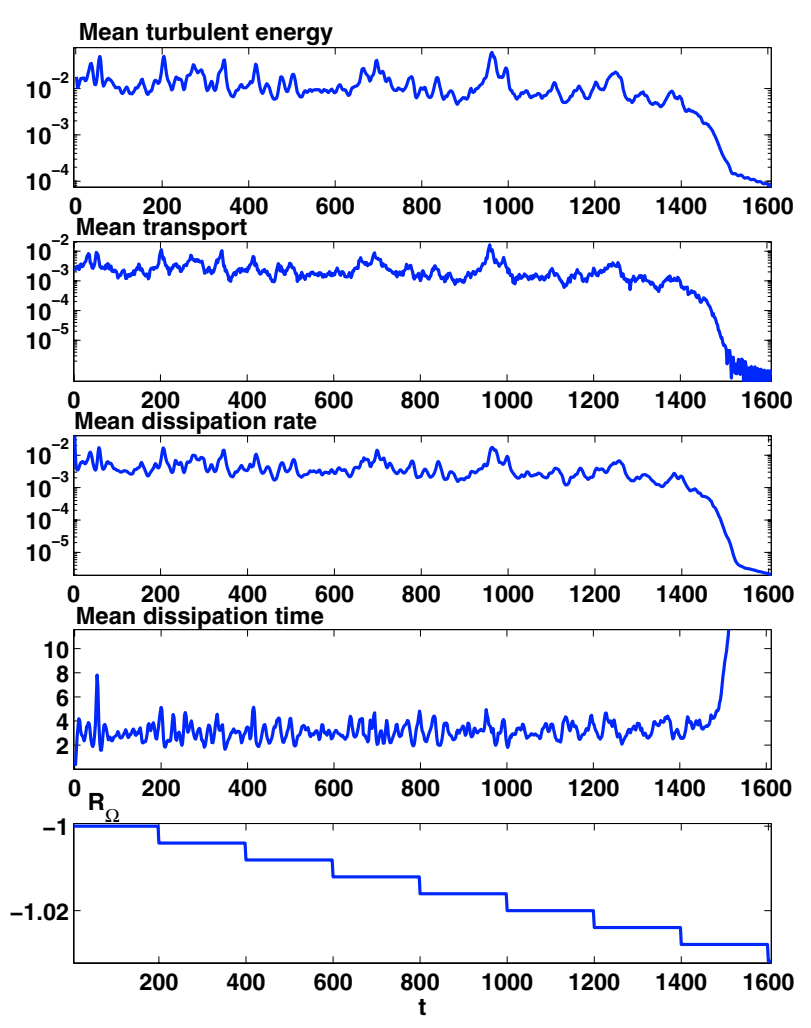

Fig. 6. Time evolution of a $64^{3} \mathrm{Re}=12000$ anticyclonic flow as computed by our Fourier code. Panel description is identical to Fig. 3.

example Fig. 6, we find the last turbulent point for $\mathrm{Re}=12000$ at $R_{\Omega}=-1.024$.

As for cyclonic rotation, the last turbulent points for anticyclonic rotation are plotted in Fig. 7 and the mean transport in Fig. 8. Error bars are added in Fig. 7 to help assessing the significance of the various fits performed, as they will be used later on. On the lower bound of these bars, turbulence is not maintained with certainty whereas the contrary is true for at least 200 shear times at the upper bound. Therefore, the actual transition Reynolds number is bracketed by the error bar.

Recalling that $R_{\Omega}=-2 / q$ with $\Omega(r) \propto r^{-q}$ and that $R_{\Omega}=-1$ corresponds to a constant specific angular momentum distribution in cylindrical flows, the largest rotation number reached here $\left(R_{\Omega}=-1.032\right)$ corresponds to $q=1.94$; this is quite consistent with the results shown in Fig. 1 of Hawley et al. (1999), except for the crucial fact that the resolution and Reynolds number dependence are now quantified. The reason why such high Reynolds numbers are accessible with our relatively low resolutions is discussed in Sect. 4.4. For the time being, let us comment a bit further on the information encoded in Fig. 7, which shows that Reynolds number and resolution are different, albeit related control parameters. We will focus on the Fourier code data for definiteness. Consider the $32^{3}$ data, for example. For $\left|R_{\Omega}\right|<1.016$, the transition Reynolds number agrees with the one found at higher resolution. However, increasing the Reynolds number above $\sim 6000$ produces a loss of turbulence at the same rotation number independently of the Reynolds number, whereas this is not true at higher resolutions. This implies that the physics is not faithfully represented at this resolution for $\operatorname{Re}>6000$ and $R_{\Omega}>1.016$. 


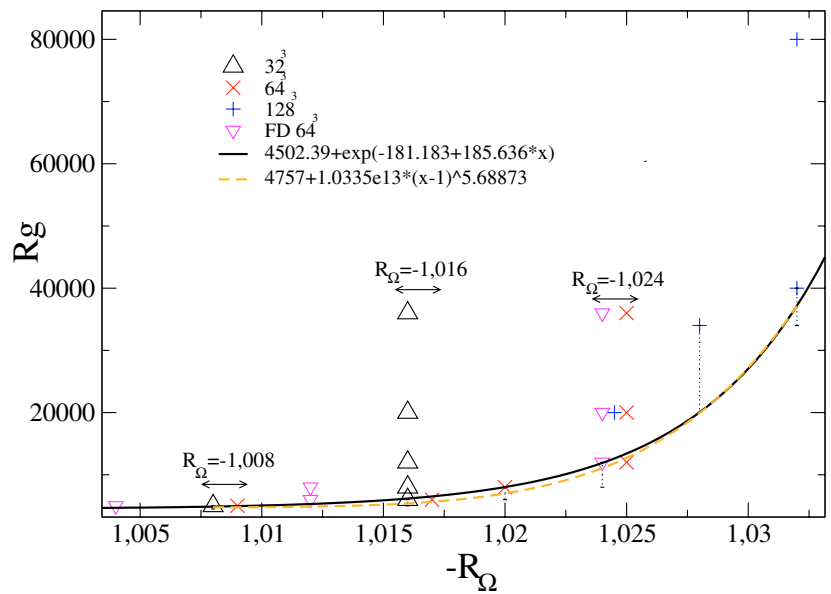

Fig. 7. Transition Reynolds number $R g$ as a function of the Rotation number $R_{\Omega}$, and related analytical fits, with different resolutions and codes for shearing-sheet boundary conditions (anticyclonic side). All plots were computed using our Fourier code except FD (finite difference) which uses our ZEUS-like code. Note that the $x$-axis is inverted with respect to Fig. 2. Symbols along the fitted lines correspond to resolved simulations; vertically aligned symbols indicate the limiting rotation number that can be reached at a given resolution and mostly correspond to unresolved simulations. For the sake of clarity, symbols which sit on top of each other have been slightly displaced along the $R_{\Omega}$ axis; this is indicated by the arrows and the related values of $R_{\Omega}$.

This is the most important point to note here: two different regimes of transition from turbulent to laminar are displayed in this figure. The first (corresponding to the various fitting curves) is the correct, resolution independent and Reynolds dependent transition. The second (apparent as the various vertically aligned points at a given resolution) is an incorrect, Reynolds independent and resolution limited transition. Note that the points belonging to both this vertical line and the laminar-turbulent line are still resolved, though, as shown in Sect. 4.4.2. The meaning of the behavior displayed in Fig. 7 is further discussed in Sect. 4.1, and its implications in Sects. 4.2 and 4.4 .

Comparing Figs. 4 and 7, we remark that the dependence of the transition Reynolds number $R g$ on the "distance" to marginal stability in rotation number $\left|R_{\Omega}-R_{\Omega}^{ \pm}\right|$is considerably stiffer on the anticyclonic side than on the cyclonic one. This has important implications that will be discussed in the next section. Conversely, the turbulent momentum transport is very similar to the one found for the cyclonic side ${ }^{5}$, as shown in Fig. 8

$\left\langle v_{x} v_{y}\right\rangle \simeq \frac{5.5}{R g-3000}(S d)^{2}$.

The constant in the denominator differs from the one found on the cyclonic side. This reflects the difference of transition Reynolds number at the two marginal stability limits. For large enough Reynolds number, one find $\left\langle v_{x} v_{y}\right\rangle \simeq 5.5 / \mathrm{Rg}$, which

\footnotetext{
${ }^{5}$ Figure 8 is noisier than its cyclonic counterpart. This is a consequence of the larger turbulent fluctuations observed in anticyclonic flows. Longer integrations time-scale would have been required to improve the statistics.
}

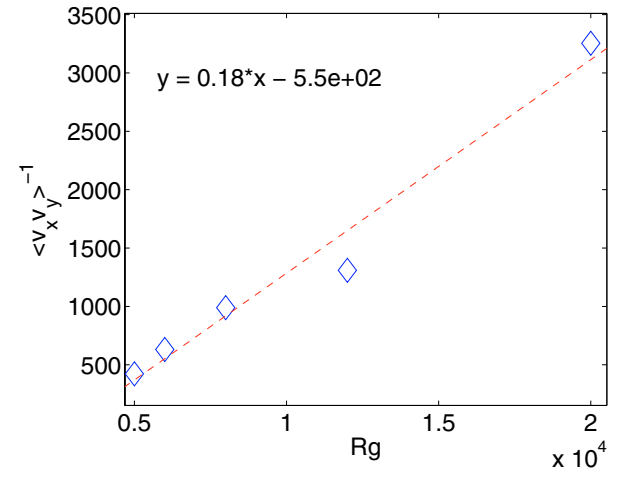

Fig. 8. Mean transport as a function of critical reynolds number on the anticyclonic side (normalized by $S^{2} d^{2}$ ).

corresponds to the asymptotic relation found on the cyclonic side (see Sect. 4.1 for a discussion of the possible origin of this behavior). This indicates that this relation is very robust for subcritical flows, far enough from the supercritical transition limit.

\section{Discussion}

Our results are at variance with both the point of view advocated by Balbus et al. (1996) and Hawley et al. (1999) (absence of subcritical turbulence), and Richard \& Zahn (1999) and Hersant et al. (2005) (efficient transport due to subcritical turbulence). This is further investigated in this section. We shall first present some phenomenological background material which helps to understand the physical origin and meaning of the results presented in the previous section. Then, we shall respectively discuss the implications of our results for Keplerian flows (Sect. 4.2), the stabilizing role of the Coriolis force in subcritical flows (Sect. 4.3), and the relation between Reynolds number and resolution (Sect. 4.4); these last two items have been highly controversial in the past decade. Section 4.4 also discusses the relation of these results with the scale-invariance argument of Balbus (2004). Finally the influence of the nature of the adopted boundary conditions and aspect ratio on our results is the object of Sect. 4.5, as well as their relation to fluid dynamics experiments. Note also that the discussion of the boundary conditions helps quantifying possible biases introduced by the sheering sheet boundary conditions with respect to actual disk physics. The reader interested only in the astrophysical implications of our results may focus in Sect. 4.2.

\subsection{Some aspects of subcritical turbulence phenomenology}

The phenomenology of subcritical turbulence has been discussed in Longaretti (2002) and Longaretti \& Dauchot (2005). Some directly relevant aspects for our present purpose are presented here (and clarified where needed).

Turbulent transport is often quantified in terms of a turbulent viscosity. As this description has been criticized in the past, a brief discussion of its use here might be useful. First, note that, in scale-free systems such as the ones studied here (the 
only scale present being the simulation box size), one can always assume that

$\left\langle v_{x} v_{y}\right\rangle=v_{\mathrm{t}} S$

as this only amounts to defining a turbulent viscosity $v_{\mathrm{t}}$ such that this relation is satisfied. In any case, as the source of turbulence is the shear, the Reynolds stress $\left\langle v_{x} v_{y}\right\rangle$ must be some function of the shear $S$, which cancels when the shear cancels.

Now, $v_{\mathrm{t}}$ has the dimension of a length times a velocity, so that one must therefore have, in our simulations,

$v_{\mathrm{t}}=\alpha S d^{2}$,

as $S d$ and $d$ are the only dimensional quantities with the right dimensionality introduced in the problem.

$\alpha$ is a Shakura-Sunyaev-like parameter. It is a dimensionless quantity, and can therefore only depend on the dimensionless quantities ${ }^{6}$ characterizing the problem at hand, namely the Reynolds number $R e$ and the rotation number $R_{\Omega}$ (i.e., the shear dependence of $\alpha$ can only appear through the ratios of the shear time scale to the viscous and the rotation time scales):

$\alpha \equiv \alpha\left(\operatorname{Re}, R_{\Omega}\right)$

The results of Sect. 3 suggest that, quite remarkably, $\alpha$ depends only on $R_{\Omega}$ through the transition Reynolds $R g$, and not (or little) on $\mathrm{Re}$, in subcritical flows. The origin of this behavior can be understood in the following way (Longaretti 2002).

A sheared flow is out of global thermodynamical equilibrium, and tries to restore this equilibrium by transporting momentum across the shear. A subcritical flow has only two means at its disposal to achieve this purpose: laminar and turbulent transport. It will tend to choose the most efficient one under any given set of conditions ${ }^{7}$, i.e. at given $\mathrm{Re}$ and $R_{\Omega}$. The subcritical turbulent transport will exceed the laminar one when $v_{\mathrm{t}} \gtrsim v$. Right at the laminar-turbulent threshold, $\operatorname{Re} \sim R g$ and $v_{\mathrm{t}} \sim v$. This implies that

$\alpha \sim \frac{v}{S d^{2}} \sim \frac{1}{R g}$

Now, what does happen at Reynolds numbers Re larger than the transition Reynolds number $R g$ ? To answer this question, it is useful to have in mind some idealized, qualitative picture of the situation in wave-number space. Such a picture is proposed in Fig. 9, and constitutes a reasonable working hypothesis. It is reasonably well-supported by our current knowledge of the plane Couette flow turbulent self-sustaining process and of inertial spectra, as well as by the spectral analysis of some of our simulations presented and discussed in Sect. 4.4.2.

In this picture, the large scales are occupied by the selfsustaining mechanism. All scales in this domain are expected to be coherent in phase, and interactions between large and small

\footnotetext{
${ }^{6}$ Actually, in principle, $\alpha$ depends also on the aspect ratio of the simulation, and on the nature of the boundary conditions. As these are not varied in the results discussed on the basis of the phenomenology described here, this dependency is ignored for simplicity.

7 Note that this does not imply that the momentum transport is absolutely maximized.
}

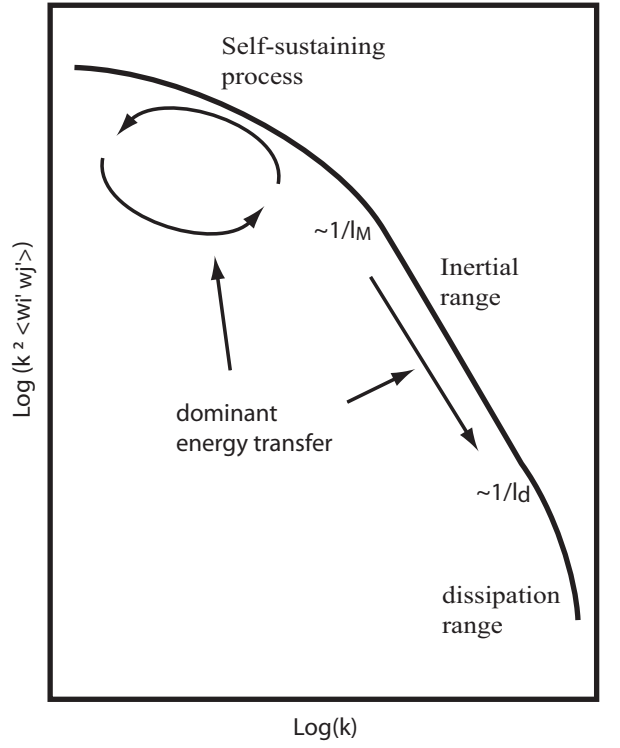

Fig. 9. Proposed sketch of the idealized energy spectrum in a turbulent shear flow. Arrows indicate the energy flow through mode coupling. The length-scales $l_{M}$ and $l_{\mathrm{d}}$ correspond to the top of the inertial range (assumed identical to the bottom of the self-sustaining range for simplicity) and the top of the dissipation range. Scales are assumed to be normalized to the box simulation size $d$, and anisotropy is ignored in this sketch (see text for details).

scales occur both ways ${ }^{8}$. The intermediate range is the inertial range of turbulence; scales have no phase coherence, energy cascades to smaller scales at a constant rate, provided by the self-sustaining mechanism (as part of the mode coupling taking place in the self-sustaining mechanism range of scales occurs with the inertial range). The smallest range represents the viscous dissipation scales. The existence of the self-sustaining process scales, their properties, and their influence on the inertial range (energy input and anisotropy) is the distinctive feature of shear turbulence with respect to the more commonly known and studied forced isotropic turbulence.

In such a picture, increasing the Reynolds number almost exclusively results in an increase of the inertial range, which is essentially vanishing at the transition Reynolds number. This should have little effect on the turbulent transport (whereas, on the contrary, the laminar transport becomes smaller and smaller when increasing the Reynolds number).

Indeed, we have first checked that this is case in nonrotating Couette plane flows, where the self-sustaining mechanism is identified (Hamilton et al. 1995): the transport is almost completely determined dominated by the mechanism rolls and streaks. Furthermore, in our simulations, we have computed the contribution of each length scale to the total transport $\left\langle v_{x} v_{y}\right\rangle$. First one should note that in Fourier space (in 1D for simplicity):

$\left\langle v_{x} v_{y}\right\rangle=\sum_{n=0}^{N-1} \tilde{v_{x}}\left(k_{n}\right) \tilde{v_{y}^{*}}\left(k_{n}\right)$.

8 This is the case in particular for the non-rotating plane Couette self-sustaining mechanism (Waleffe 1997). 


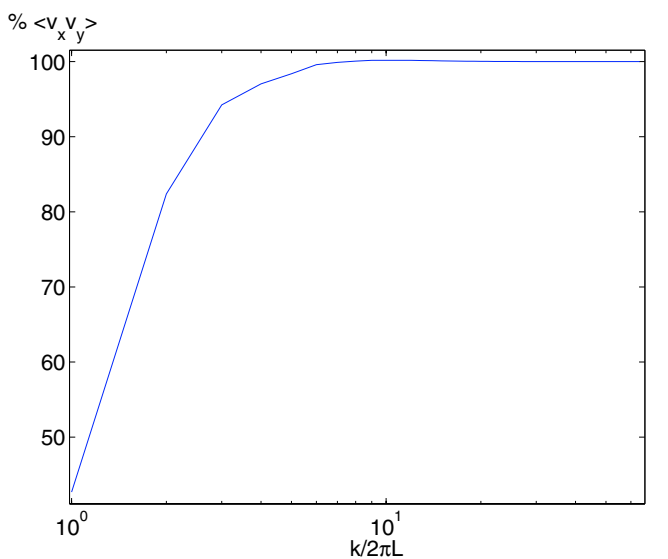

Fig. 10. Example of the cumulative sum contribution of each scale length to the mean transport, starting from $k_{x}=0$ in the $x$ direction: $99 \%$ of the transport comes from $k_{x}<10$ scales. From a $128^{3}$ simulation, $\operatorname{Re}=20000, R_{\Omega}=-1.020$.

Therefore, the contribution to mean transport of the wavelength $k_{n}$ is found to be $2 \Re\left(\tilde{v_{x}}\left(k_{n}\right) \tilde{v_{y}^{*}}\left(k_{n}\right)\right)$, since $k_{n}$ and $k_{N-n}$ represents the same physical wavenumber. This simple result can be used in 3D by averaging the transport over 2 directions (in physical space) and by computing the Fourier transform in the remaining direction; this procedure is sufficient for our purpose here. The resulting cumulative Fourier sum, starting from $n=0$ is illustrated on an example in Fig. 10 to quantify which scales dominate the transport. In this example, the resulting spectral analysis is plotted in the $x$ direction for a $128^{3}$ anticyclonic flow with $R_{\Omega}=-1.024$ and $\operatorname{Re}=20000$, showing that more than $99 \%$ of the transport comes from scales larger than $1 / 10$ of the box size; this range corresponds to the length-scales of the self-sustaining process (see Sect. 4.4.2). Similar results are found for spectral analyzes in the $y$ and $z$ directions, consistently with the picture discussed here. This is expected anyway if the inertial spectrum is Kolmogorovian, as confirmed from the spectral analysis of Sect. 4.4.2 ${ }^{9}$.

There are two loose ends in this discussion. First, hysteresis is usually experimentally observed in subcritical transitions to turbulence: the measured transition Reynolds number is higher when moving "up" from the laminar to turbulent states than when moving "down" from the turbulent to laminar ones. This suggests that the laminar-turbulent boundary is separated by some sort of barrier in the appropriate phase-space (defined, e.g., by the amplitudes and phases of the Fourier modes). This (along with the fact that the arguments developed here apply only in order of magnitude) may well explain the existence of the constant of order 5 that one finds in Eqs. (19) and (20) with respect to Eq. (24). Secondly, the arguments presented here ignore the existence of marginal stability thresholds. This, as pointed out in Sects. 3.3 and 3.4, may explain the presence of the constant at the denominator of these relations, as the equivalent global subcritical transition Reynolds number that one

\footnotetext{
9 The true nature of the inertial spectrum might be affected by the anisotropy generated by the the shear and the Coriolis force, but these anisotropies must become negligible at small scale, due to the shorter and shorter eddy turnover time.
}

can define in the supercritical regime is orders of magnitude smaller than in the subcritical regime.

To conclude, let us point out the relation of this picture with the numerical results presented in Fig. 7. The fact that higher resolutions are required to faithfully represent the physics at higher rotation numbers indicates that the ratio $d / l_{M}$ increases with rotation number. Indeed, if the resolution is too low, so that the relative scale $l_{M} / d$ is not resolved, the energy transfer loop represented in Fig. 9 cannot take place, and turbulence is not self-sustained. Furthermore, at the transition Reynolds number, the inertial spectrum is nearly inexistent, as pointed out above, and $l_{M} \sim l_{\mathrm{d}}$. Consequently, the most critical scale ratio in this problem is expected not to be the Kolmogorov one, but the self-sustaining mechanism one $\left(d / l_{M}\right)$.

\subsection{Implications for Keplerian flows}

Actual disks are vertically stratified, whereas stratification is ignored in our experiments. Stratification provides us with a local macroscopic scale (the disk scale height $H$ ). With appropriate provisos relating to the possible stabilizing or destabilizing role of stratification ${ }^{10}$, one can tentatively identify this scale height with our simulation box size: $H=d$. This assumption is made throughout this section. In the same way, the Shakura-Sunyaev $\alpha_{S S}$ parameter is defined such that $v_{\mathrm{t}}=\alpha_{S S} c_{\mathrm{s}} H \simeq \alpha_{S S} \Omega H^{2}$. Equation (22) then implies that $\alpha_{S S}=2 \alpha /\left|R_{\Omega}\right| \simeq \alpha$ (the last equality holds within a factor of order unity for the rotation number range of interest in this work).

Using the numerical results shown in Figs. 7 and 8, one can deduce a few properties of Keplerian flow subcritical shear turbulence, based on various conservative extrapolations of our numerical data. First, the transition Reynolds number $R g$ dependence on the rotation number $R_{\Omega}$ is well-fitted by a power or an exponential law. Using these laws, one can get a first set of estimates of the transition Reynolds number for Keplerianlike flows $\left(R_{\Omega}=-4 / 3\right): R g=1.1 \times 10^{10}$ and $R g=1.3 \times 10^{26}$, respectively. The last estimate leads to the absence of subcritical turbulence in accretion disks whereas the first one allows for its existence ${ }^{11}$. Secondly, let us note that, for both cyclonicity, the Coriolis force induces a steeper and steeper increase of the transition Reynolds number when moving away from the marginal stability boundary. This suggests that one can find a lower bound for $R g$ by linearly extrapolating the power law fit beyond the last known point $\left(R_{\Omega}=-1.032\right)$. One find this

${ }^{10}$ If stratification is destabilizing, the momentum transport induced by the resulting convective motions is in the wrong direction, as recalled in the introduction, and must be counterbalanced by another process; ignoring stratification in this case therefore makes life easier for this other process (here, subcritical turbulence). If stratification is stabilizing, this also most likely results in an increased difficulty in finding the transition to turbulence, and a related increase in the transition Reynolds number. These arguments suggest that ignoring the dynamical stratification altogether maximizes the overall outwards transport in our problem.

11 We assume that accretion disk Reynolds numbers lie between $10^{10}$ and $10^{15}$ for definiteness. The Reynolds number definition used in this evaluation is $\operatorname{Re}=S H^{2} / v$ where $H$ is the local disk scale height, consistently with the $H=d$ identification made earlier. 
Table 1. Extrapolated transition Reynolds numbers, values of $\alpha$, and required simulations resolution, for Keplerian flows, under various assumptions (see text for details).

\begin{tabular}{lllll}
\hline \hline & exponential & power-law & cyclonic & linear \\
\hline$R g$ & $1.3 \times 10^{26}$ & $1.1 \times 10^{8}$ & $2 \times 10^{7}$ & $1.8 \times 10^{6}$ \\
$\alpha$ & n/a & $5 \times 10^{-10}$ & $2.6 \times 10^{-7}$ & $3.1 \times 10^{-6}$ \\
$(d / \delta)^{3}$ & n/a & $7000^{3}$ & $3000^{3}$ & $900^{3}$ \\
\hline
\end{tabular}

way $R g^{\min }=1.8 \times 10^{6}$. As a final hypothesis, one may envision that the $R g\left(R_{\Omega}\right)$ relation would be more or less symmetric with respect to $R_{\Omega}=0$ if there were no supercritical domain. This would explain why the actual relation of Fig. 7 is so steep: in this picture, the system tries to reach back as fast as possible the high values of transition Reynolds number expected from this hypothetical symmetry, after which the Reynolds dependence with rotation number would be much less steep. Under this assumption the expected transition Reynolds number for Keplerian flows would be $R g=2 . \times 10^{7}$ (a power-law fit of the cyclonic data has been used in this extrapolation).

This information is summarized in Table 1, along with the corresponding values of $\alpha$, obtained from the asymptotic relation $\alpha=\left\langle v_{x} v_{y}\right\rangle=5.5 / R g$ found for cyclonic and anticyclonic flows in the previous section. The last line shows the resolution required to successfully simulate Keplerian flow turbulence, for the various Reynolds numbers (see Sect. 4.4.1). One sees that even the most optimistic $\alpha$ bound $\left(\alpha_{\max }=3.1 \times 10^{-6}\right)$, obtained with the linear extrapolation, is substantially smaller than the values required in astrophysical accretion disks (as summarized, e.g., in Papaloizou \& Lin 1995). Note finally that, even without any extrapolation, our results exclude subcritical turbulent transport at the $\alpha \simeq 3 \times 10^{-4}$ level.

\subsection{Role of the Coriolis force in uniform shear flows}

Two different but related issues have been raised in the literature concerning the role of the Coriolis force in subcritical systems.

First, for linearly stable flows, Balbus et al. (1996) point out that the Coriolis force plays a conflicting role in Eqs. (4) and (5). More precisely, they make the following point: as $S\left\langle v_{x} v_{y}\right\rangle>0$ for turbulence to exist (see Sect. 2.2), the terms in which the shear $S$ has been factored out in these equations have opposite signs for linearly stable flows, while they have the same sign for linearly unstable flows (note that this is true independently of the flow cyclonicity). They conclude from this that a stabilizing rotation prevents turbulence to show up in subcritical shear flows, except possibly in the vicinity of marginal stability. Somewhat relatedly, the recent Reynolds stress-closure model of Ogilvie (2003) and Garaud \& Ogilvie (2005) predicts relaminarization for large enough deviations from the marginal stability limit. In particular, for the authors' standard choice of parameters, it predicts relaminarization for $R_{\Omega} \sim 0.2$ for cyclonic rotation. However, as can be seen in Fig. 4, both the Balbus et al. (1996) argument and the Garaud \& Ogilvie (2005) result conflict with our simulations: subcritical turbulence is maintained away from marginal stability on the cyclonic side, at least up to $R_{\Omega} \simeq 0.3$. Note that we could have pushed the search for transition to turbulence beyond what is shown on this graph, especially by using higher resolutions, but did not do it due to computer resources limitations. As discussed in the next subsection, the absence of turbulence in the Keplerian flow simulations of Balbus et al. (1996) and Hawley et al. (1999) is a problem of resolution.

The second issue relates to the asymmetry between cyclonic and anticyclonic rotation. The stress-closure model just mentioned depends on the rotation number only through the combination $R_{\Omega}\left(R_{\Omega}+1\right)$ which implies a symmetry with respect to $R_{\Omega}=-1 / 2$. This symmetry is clearly violated by our numerical results (compare Figs. 4 and 7), a point which requires some comments.

First, note that the linearized Navier-Stokes Eq. (1) exhibits this symmetry for perturbations with vanishing pressure variation $(\delta \pi=0)$. In this case, the linearized equation can be written:

$\frac{\partial \boldsymbol{w}}{\partial t}=S \cdot y \frac{\partial \boldsymbol{w}}{\partial x}+S \cdot\left(\left(R_{\Omega}+1\right) w_{y} \boldsymbol{e}_{x}-R_{\Omega} w_{x} \boldsymbol{e}_{y}\right)+v \Delta \boldsymbol{w}$.

The cyclonic-anticyclonic symmetry appears when exchanging the $x$ and $y$ directions. Indeed, upon the following change of variables:

$R_{\Omega}^{\prime}=-R_{\Omega}-1$,

$w_{x}^{\prime}=w_{y}, \quad \boldsymbol{e}^{\prime}{ }_{x}=\boldsymbol{e}_{y}$,

$w_{y}^{\prime}=w_{x}, \quad \boldsymbol{e}^{\prime}{ }_{y}=\boldsymbol{e}_{x}$,

$w_{z}^{\prime}=w_{z}, \quad \boldsymbol{e}_{z}^{\prime}=\boldsymbol{e}_{z}$,

so that

$\boldsymbol{w}^{\prime}=w_{x}^{\prime} \boldsymbol{e}_{x}^{\prime}+w_{y}^{\prime} \boldsymbol{e}_{y}^{\prime}+w_{z}^{\prime} \boldsymbol{e}_{z}^{\prime}$

$=w$,

the form of Eq. (26) should be invariant, which is indeed the case:

$\frac{\partial \boldsymbol{w}^{\prime}}{\partial t}=S \cdot y \frac{\partial \boldsymbol{w}^{\prime}}{\partial x}+S \cdot\left(\left(R_{\Omega}^{\prime}+1\right) w_{y}^{\prime} \boldsymbol{e}^{\prime}{ }_{x}-R_{\Omega}^{\prime} w_{x}^{\prime} \boldsymbol{e}_{y}\right)+v \Delta \boldsymbol{w}^{\prime}$.

This symmetry can also be extended to compressible motions by adding $\delta \pi^{\prime}(x, y, z)=\delta \pi(y, x, z)$ to the list of change of variables.

Because the perturbations defining the linear stability limit also exhibit this symmetry (Appendix A), it has often been assumed in closure-stress models in the past. However, this is not a symmetry of the full Navier-Stokes equation (Speziale \& Mhuiris 1989; Speziale 1991; Salhi \& Cambon 1997), nor of the $\boldsymbol{\nabla} \cdot \boldsymbol{w}=0$ equation). This is also apparent in a direct inspection of the structure of simulated turbulent flows. The $R_{\Omega}=0$, wall-bounded turbulent flows contain large streamwise rolls living for about a hundred shear times (Hamilton et al. 1995). We have also found rolls more or less aligned in the streamwise direction in our $R_{\Omega}=0$ shearing sheet simulations, although we did not try to precisely quantify their survival time. Furthermore, at the anticyclonic marginal stability limit $\left(R_{\Omega}=-1\right)$, we did observe sheared shearwise rolls (i.e. rolls in $y$ direction) in our simulations, as one might expect 
from the symmetry of the linearized Navier-Stokes equation. The anticyclonic roll survival time is observed to be rather short compared to their cyclonic counterpart, as they are tilted by the shear and loose their coherence in a few shear times at most. This roll lifetime is the main difference we found between the cyclonic and anticyclonic side. This is related to the fact that a streamwise roll does not reduce the shear on the anticyclonic subcritical domain (in opposition to the cyclonic one).

In any case, we have found turbulence away from the marginal stability limit in cyclonic flows, and the symmetry with respect to $R_{\Omega}=-1 / 2$ is violated both in our simulations, and in supercritically rotating shear flow turbulence (see, e.g., Salhi \& Cambon 1997 and references therein). This make the predictions of the stress-closure model of Ogilvie (2003) and Garaud \& Ogilvie (2005) unreliable in both subcritical and supercritical flows.

\subsection{Resolution, effective Reynolds number and scale invariance}

The results of Sects. 3.3 and 3.4 involve fairly high Reynolds numbers, and one might ask if our simulations are resolved enough in these regimes. This question has a priori two different aspects, as one can guess from Fig. 9: resolving the selfsustaining process smallest relative scale $d / l_{M}$, and resolving the relative dissipation scale $d / l_{\mathrm{d}}$.

For the problem considered in this paper, resolving the first scale is a sine qua non condition: if it is not satisfied, turbulence does not show up, independently of the simulation Reynolds number, because the required scale coupling shown in Fig. 9 for the self-sustaining process to exist cannot take place. This shows up in Fig. 7 as the vertical transition limit from turbulent to laminar that we obtained for any given resolution, for large enough Reynolds numbers.

Resolving the dissipation scale is important to ascertain that direct numerical simulations such as the ones performed here are not biased by (the presence or absence of) numerical dissipation, and this issue is often raised in the fluid mechanics literature. For the time being, we note that, at the transition Reynolds number, the inertial domain should be non-existent or extremely reduced, so that $l_{M} \simeq l_{\mathrm{d}}$ and both resolution requirements should be directly related (this point, used in Sect. 4.4.1 is justified in Sect. 4.4.2). We can therefore consider that the "effective Reynolds number" $\mathrm{Re}_{\text {eff }}$ of our simulations is the largest transition Reynolds number $R g$ correctly determined at a given resolution ${ }^{12}$, as discussed in Sect. 3.4.

Note that this effective Reynolds number is problemdependent: the self-sustaining process qualitative and quantitative characteristics both depend on the considered problem; furthermore, in simulations of isotropic turbulence, the selfsustaining process is absent, and replaced by a forced amplitude of the largest Fourier modes, so that the effective Reynolds number in this case is the one related to the dissipation scale.

\footnotetext{
${ }^{12}$ With all the provisos discussed in Sect. 3.2 about the role of the choice of the initial conditions and turbulence minimal survival lifetime.
}

Let us now examine the two requirements mentioned above in more detail.

\subsubsection{Resolving the self-sustaining process}

First, we would like to qualitatively comment on the difference of resolution requirements between cyclonic and anticyclonic flows.

As discussed in Sect. 4.5, the nature of the shearwise boundary condition has apparently only a small influence on the results; this is exemplified by the similar transition Reynolds numbers found in our simulations and in experiments on rotating shear flows (see Fig. 14). This suggests that at least some of the characteristics of the self-sustaining process of non-rotating plane Couette flows are relevant here. At the cyclonic marginal stability limit, this self-sustaining process has a time-scale $t_{\mathrm{SSP}} \sim 100 S^{-1}$ (Hamilton et al. 1995; Waleffe 1997). The requirement that, at the transition Reynolds number, the viscous time scale at scale $l_{M}$ exceeds $t_{\mathrm{SSP}}$ reads $l_{M}^{2} / v \gtrsim 100 S^{-1}$, i.e., $l_{M} / d \lesssim(100 / R g)^{1 / 2} \sim 1 / 4$ for $R g \sim$ 1500 (Longaretti \& Dauchot 2005). This probably explains why the resolution requirement is so low on the cyclonic side. Conversely, we have mentioned at the end of the previous subsection that rolls (which are an apparently ubiquitous ingredient in subcritical turbulence) do not survive more than a few shear times in anticyclonic flows. Therefore, the anticyclonic self-sustaining process time-scale cannot exceed a few shear times as well, whatever its nature. The same reasoning as the one exposed above leads to $l_{M} / d \lesssim$ a few $(1 / R g)^{1 / 2} \sim$ a few $\times 1 / 70$, an already much more demanding constraint. It is obviously related to the larger transition Reynolds number found at the anticyclonic marginal stability, compared to the cyclonic one.

As mentioned several times already, the self-sustaining process is identified and understood only at the cyclonic marginal stability limit in wall-bounded Couette flows. Consequently, it is difficult to explain why the resolution demand grows so much faster with rotation number "distance" to marginal stability for anticyclonic flows than for cyclonic ones. However, we speculate that this is connected to the fact the rotation time scale is only a fraction of $S^{-1}$ for cyclonic flows, whereas it always exceeds $S^{-1}$ for anticyclonic ones.

Next, let us try to quantify the resolution that would be needed to successfully simulate Keplerian flows. The phenomenology of subcritical turbulence developed by Longaretti (2002) predicts that $d / l_{M} \sim R g^{1 / 2}$ and $\left\langle v_{x} v_{y}\right\rangle \propto 1 / R g$. This phenomenology implicitly assumes that the relevant time-scale of the self-sustaining process is $\sim S^{-1}$, so that it would need to be modified to be applied to cyclonic flows, but it should be adequate for anticyclonic ones, with appropriate modifications. In particular, we have already pointed out in Sect. 4.1 that the last relation needs to be amended into $\left\langle v_{x} v_{y}\right\rangle \propto 1 /\left(R g-R_{\mathrm{c}}\right)$ with $R_{\mathrm{c}} \simeq 3000$ on the anticyclonic side. This suggests that

$\frac{\delta}{d} \simeq \frac{\gamma}{\left(R g-R_{\mathrm{c}}\right)^{1 / 2}}$

is the appropriately generalized scale relation ( $\delta$ being the smallest scale accessible to the simulation, i.e., the resolution). 
Table 2. Resolution, effective Reynolds number and $\gamma$ factor for the Fourier code on the anticyclonic side.

\begin{tabular}{lll}
\hline \hline$(d / \delta)^{3}$ & $\operatorname{Re}_{\text {eff }}$ & $\gamma$ \\
\hline $32^{3}$ & 6000 & 1.71 \\
$64^{3}$ & 12000 & 1.48 \\
$128^{3}$ & 38000 & 1.46 \\
\hline
\end{tabular}

Table 2 gives the values of $\gamma$ and $\operatorname{Re}_{\mathrm{eff}}$ for the three different resolutions of our anticyclonic simulations.

Although the statistics is a little poor to draw firm conclusions, it appears that $\gamma$ is nearly constant compared to the variations in both resolution and transition Reynolds number, and our simulations are therefore consistent with Eq. (28). The resolution needed to simulate Keplerian flows has been computed based on the estimate Eq. (28), with $\gamma=1.5$ (the $R_{\mathrm{c}}$ correction has little influence on these estimates). The results are shown in Table 1. For comparison purposes, note that the largest turbulence simulation ever performed was $4000^{3}$, but was not run for hundreds or thousands of dynamical times. Although the results gathered here are probably only indicative, as they are based on guess work, they strongly suggest that simulating subcritical turbulence in Keplerian flows is beyond present day computer capabilities, and support the idea that the subcritical Keplerian flows simulations performed to date were limited by numerical resolution, as suggested by Longaretti (2002).

\subsubsection{Resolving the dissipation scale}

In statistically steady turbulence, the dissipation scale can be defined from the balance between input and dissipation described by Eq. (8). The energy input is provided by $S\left\langle v_{x} v_{y}\right\rangle$. The Fourier analysis of this quantity is shown in Fig. 10, and is dominated by the large scales. Conversely, the Fourier content of $\epsilon$, Eq. (7), is dominated by the small scales (large $k$ ), comparable to the dissipation scale, as illustrated below.

Resolving the dissipation scale is important with Fourier codes in order to prevent energy accumulation at the smallest scales, which may bias the results, or lead to code $\operatorname{crash}^{13}$.

The general definition of the dissipation wavelength $k_{\mathrm{d}}$ follows from the evaluation of Eq. (7) in Fourier space:

$\epsilon=2 v \int_{0}^{k_{\mathrm{d}}} k^{2} E(k) \mathrm{d} k$

where it is assumed that $E(k)$ is cut-off at $k_{\mathrm{d}}$, either abruptly, or through some modelling of the dissipation range (see e.g. Lesieur 1990).

In simulations of homogeneous and isotropic turbulence, the energy input is imposed from the outside: the amplitude of the largest Fourier mode is held fixed, and Fig. 9 reduces to the inertial and dissipation range. In this context, the inertial spectrum reduces to the Kolmogorov spectrum given by:

$E_{\mathrm{K}}(k)=C_{\mathrm{K}} \epsilon^{2 / 3} k^{-5 / 3}$,

\footnotetext{
13 One may also include an hyper-viscosity term to prevent code crash, but this turned out not to be necessary.
}

where the Kolmogorov constant $C_{\mathrm{K}} \simeq 1$. Cutting off this spectrum at wavelength $k_{\mathrm{d}}$ and injecting it in the definition Eq. (29) leads to the well-known expression of the Kolmogorov wave number, $k_{\mathrm{K}}=\left(\epsilon / v^{3}\right)^{1 / 4}$. The related Kolmogorov scale (inverse of the wave number) is a largely used estimate of the dissipation scale.

In the fluid mechanics community one often requires that the Kolmogorov scale be resolved, even if the considered turbulence is not isotropic and homogeneous, as, e.g., in shear flow turbulence (see, e.g., Pumir 1996). However, in our simulations, the observed spectrum is substantially different from the Kolmogorov one, especially at the transition Reynolds number (see top panel of Fig. 12). Indeed, at the turbulent-laminar transition, one does not expect nor observe the presence of an inertial domain in the spectrum. One may therefore ask what relation the Kolmogorov scale bears to the dissipation scale of the problem.

Consider, e.g., the $32^{3}$ and $64^{3}$ energy spectra obtained at a Reynolds number $\mathrm{Re}=6000$ and a rotation number set to -1.016 . These spectra are shown on the top panel of Fig. 12. The concordance of the spectra at both $32^{3}$ and $64^{3}$ resolutions indicates that the dissipation scale in the $32^{3}$ simulation is resolved (this is consistent with the shape of the spectrum at the smallest $32^{3}$ resolved scales, much steeper than Kolmogorov). It appears that the largest distance to marginal stability $\left|R_{\Omega}+1\right|$ reliably accessible at a given resolution on the laminar-turbulent transition (as checked by higher resolution simulations) corresponds to the various vertical line of transition displayed in Fig. 7 for this resolution. In other words, the $\operatorname{Re}=6000, R_{\Omega}=-1.016$ point at $32^{2}$, and the $\operatorname{Re}=12000$, $R_{\Omega}=-1.024$ at $64^{3}$, are resolved. This feature makes us confident that the transition point determined at $128^{3}$ is the correct one, although we did not cross-check it at $256^{3}$, due to the limitations in the available computational resources.

We have thus determined the largest transition Reynolds number where the dissipation scale is confidently resolved in these anticyclonic runs at the various resolutions we have used $\left(32^{3}, 64^{3}\right.$ and $\left.128^{3}\right)$. In other words, we know the effective dissipation scale of these simulations, as it must be comparable to the largest wave number available in the simulation ${ }^{14}$ : $k_{\mathrm{d}} \simeq 3^{1 / 2} \pi N / d$, where $N$ is the resolution. Furthermore, we can compute the Kolmogorov wave number $k_{\mathrm{K}}$ for these runs, as $v=\operatorname{Re} / S d^{2}$, and as $\epsilon$ follows from Eq. (8) and the transport (e.g., with the help of the transport/transition-Reynolds-number correlation displayed in Fig. 8). The resulting ratio $R=k_{\mathrm{d}} / k_{\mathrm{K}}$ is given in Table 3.

Although the values of the ratio $R$ quoted in Table 3 are of order unity, a systematic trend seems to appear, indicating that resolving the Kolmogorov wave number is possibly not the relevant concept at the transition Reynolds number, as it is not stringent enough; nevertheless, the required resolution derived from the Kolmogorov wave number is apparently semiquantitatively correct, at least for the rotation numbers explored

\footnotetext{
14 This expression corrects a misprint in Pumir (1996) for the diagonal of a cube in Fourier space; although this largest wave number is resolved only in discrete directions, this definition is adopted here for ease of comparison with this earlier work.
} 
Table 3. Resolution, dissipation to Kolmogorov wave number ratio, and corresponding transition Reynolds number (see text for details).

\begin{tabular}{lll}
\hline \hline$N$ & $R=k_{\mathrm{d}} / k_{\mathrm{K}}$ & $R g$ \\
\hline 32 & 1.23 & 6000 \\
64 & 1.73 & 12000 \\
128 & 2.66 & 35000 \\
\hline
\end{tabular}

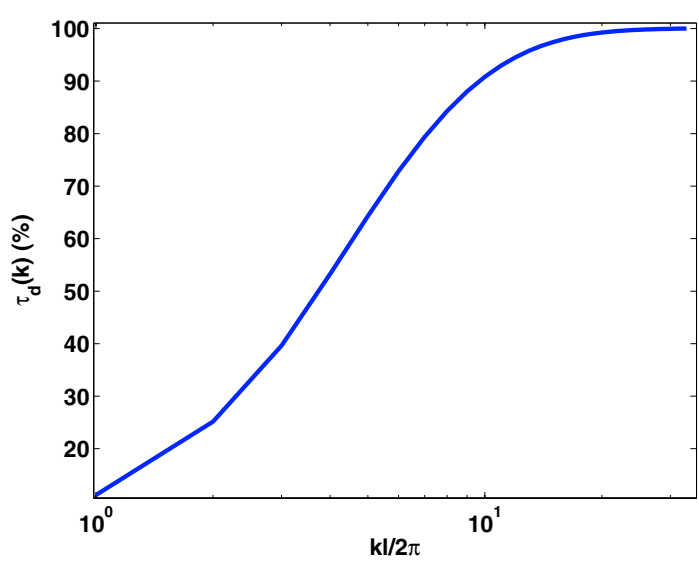

Fig. 11. Cumulative mean dissipation spectrum for a $64^{3}$ simulation at $\operatorname{Re}=6000$ for $R_{\Omega}=-1.016$.

here. Of course when going to Reynolds numbers well in excess of $R g$ at a given $R_{\Omega}$, the Kolmogorov wave number should always give the right estimate of the dissipation scale, as the inertial range becomes more and more prominent in the overall spectrum.

To conclude this aspect of the discussion, we note that both the non-Kolmogorovian shape of the spectrum at transition and the relatively small values of $\epsilon$ at the various transition Reynolds numbers used here, most probably combine in the end to explain why we can reach rather large Reynolds numbers at rather moderate resolutions.

In order to have a better grasp on which scales contribute most to the dissipation, we have computed a quantity, $\tau_{\mathrm{d}}(k)$, defined by

$\tau_{\mathrm{d}}(k)=2 v \int_{-k}^{k} \mathrm{~d} k_{x} \int_{-k}^{k} \mathrm{~d} k_{y} \int_{-k}^{k} \mathrm{~d} k_{z}\left(k_{x}^{2}+k_{y}^{2}+k_{z}^{2}\right) E\left(k_{x}, k_{y}, k_{z}\right)$.

Comparing with Eq. (29), it appears that $\tau_{\mathrm{d}}(k)$ represents the fraction of dissipation due to scales $\left|k_{x}\right|<k,\left|k_{y}\right|<k$ and $\left|k_{z}\right|<$ $k$. This quantity is plotted in Fig. 11 with the $64^{3}$ simulation spectrum. It appears that more than $95 \%$ of the total dissipation is due to $k<1 / 2 k_{\max }$ (i.e., the $32^{3}$ resolution). Also, comparing Fig. 11 with the top panel of Fig. 12 indicates that most of the dissipation comes from the part of the spectrum which is steeper than the Kolmogorov spectrum, as one would expect.

It is also instructive to examine the spectral behavior at Reynolds number larger than the transition Reynolds number, as shown in Fig. 12.

This figure displays energy spectra of the velocity deviation from the laminar flow. The rotation number is fixed
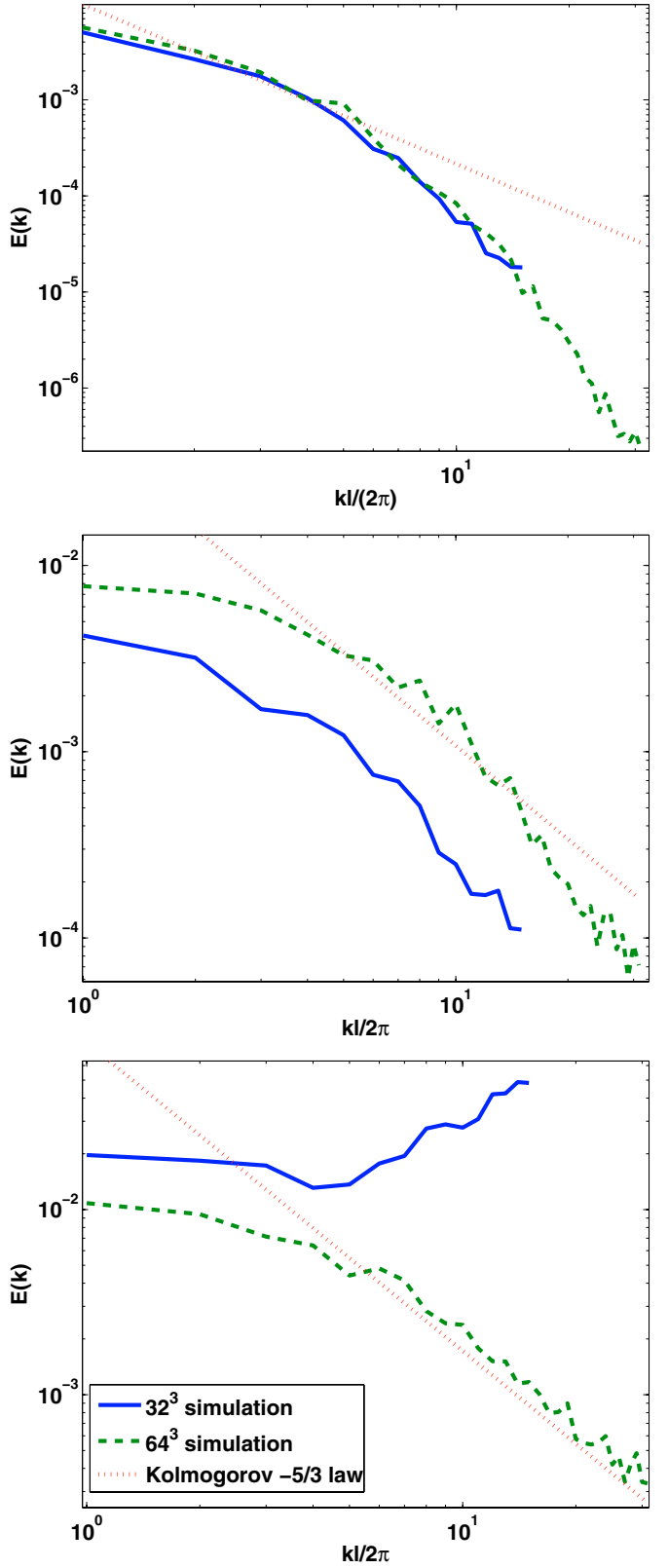

Fig. 12. Energy spectra (of the velocity deviation from the laminar flow), for two different resolutions $\left(32^{2}\right.$ and $\left.64^{3}\right)$. The rotation number is $R_{\Omega}=-1.016$ in all cases. Top panel: $\operatorname{Re}=6000$. Middle panel: $\operatorname{Re}=$ 12000. Bottom panel: $\mathrm{Re}=20000$. At this resolution only the top panel simulations are resolved, as expected. See text for discussion.

at $R_{\Omega}=-1.016$ for all spectra, and they have been averaged over a 200 shear time period to reduce the noise. From top to bottom, the Reynolds number is 6000, 12000 and 20000 respectively. The $32^{3}$ simulations are resolved only in the top panel, while the $64^{3}$ simulations should be resolved in the top two panels. Comparing the second panel with the first reveals a couple of interesting points:

- The $64^{3}$ simulation shows an extension of the spectrum, compatible with a small inertial range (this is difficult to ascertain because of the remaining noise in the simulation), while still resolving at least the top of the dissipation range, but marginally so. 


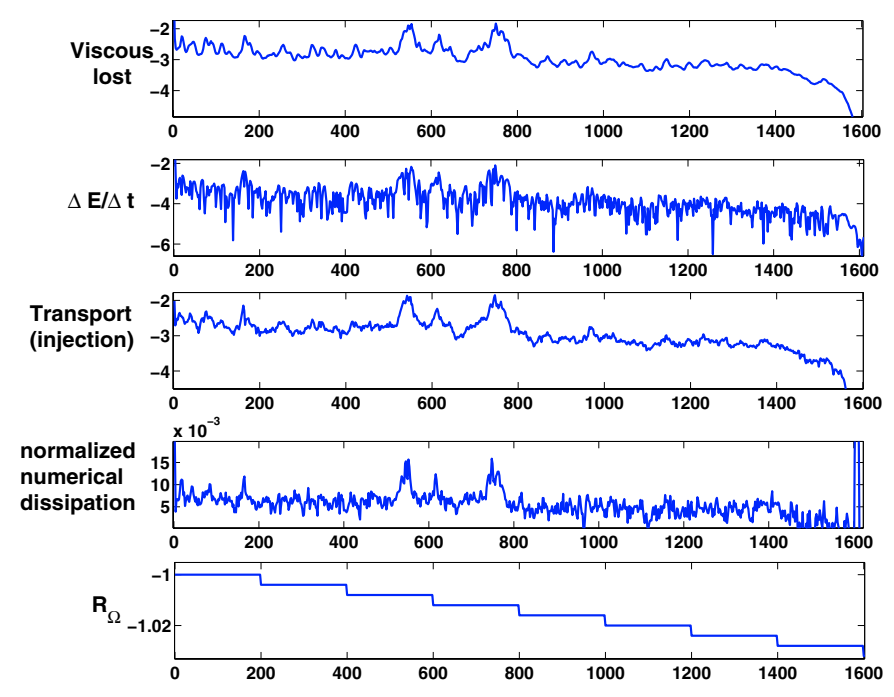

Fig. 13. Energy budget for a $64^{3} \mathrm{Re}=20000$ run with our Fourier code. Each plot represent a term in Eq. (8). The numerical dissipation is normalized by the total dissipation $\partial\left\langle\boldsymbol{w}^{2} / 2\right\rangle / \partial t-S\left\langle w_{x} w_{y}\right\rangle$. We find that numerical dissipation is about $1 \%$ of the total dissipation. NB: Vertical axis of the three first plots is in logarithmic scale.

- The $32^{3}$ simulation begins to significantly deviate from the $64^{3}$ simulation, although the trend is similar.

The third panel also displays a fairly relevant piece of information. The $64^{3}$ simulation shows both the self-sustaining mechanism scales and the inertial spectra. However, the dissipation scale does not seem to be resolved. This is not unexpected, since increasing the Reynolds number necessarily increases the inertial spectrum, and therefore decreases the dissipation scale. Apparently, the dissipation scale is probably not far off the resolved scales, so that the simulation nevertheless does not noticeably deviate from the expected behavior. But note that the $32^{3}$ simulation is clearly strongly unresolved, with energy accumulating in the small scales in order for a statistically steady equilibrium to be achieved: indeed, as this simulation resolves the self-sustaining mechanism scale, turbulence is present; however, as the smallest resolved scale is significantly larger than the dissipation scale, the spectrum must be strongly deformed to achieve a dissipation which is consistent with the energy input due to the turbulence self-sustaining mechanism.

These simulations illustrate that if the dissipation scale is not resolved, the simulated flow does not necessarily relaminarize, but the deformation of both the amplitude and shape of the spectrum most likely results in, e.g., unreliable estimates of the turbulent transport. In particular, the reliability of finite difference simulations where no viscous term is explicitly included in the code is unclear ${ }^{15}$.

On the other hand, this point is related to the fact that the numerical dissipation in a Fourier code is extremely weak, so that the deformation of the spectrum may be quite large. To compute the numerical dissipation explicitly, we have estimated its effect on the turbulent energy budget.

\footnotetext{
15 We did not further investigate this question here.
}

We plot an example of such an energy budget in Fig. 13, where all the terms in Eq. (6) are evaluated, so that the remaining difference measures the code dissipation. One should note that these plots are integrated over 2 shear times, so that they include the numerical dissipation due to the desaliazing procedure (done at each time loop) and losses from the remapping procedure (done each shear time). The presented result is generic: for all our simulations, numerical dissipation is found to be at most a few percent of the total dissipation.

In summary, we have tried as much as possible to ensure that our determination of the transition Reynolds number and turbulent transport do not suffer from lack of resolution of the dissipation scale. Note also that the results of the Fourier and finite difference codes are consistent with each other. This makes us confident that our simulations faithfully represent the relevant physics, down to and including the dissipation scale, within the relevant $\left(\mathrm{Re}, R_{\Omega}\right)$ domain determined at each resolution in Fig. 7.

\subsubsection{Shearing sheet simulations and scale invariance}

Recently, Balbus (2004) has argued that the scale invariance of the inviscid form of the Navier-Stokes equation used here makes any small scale solution exist at large scales as well. This argument seems to imply that simulations of the kind performed here should not be resolution limited. However, neither the simulations of Balbus et al. (1996), Hawley et al. (1999), the ones performed here, nor a real disk, are scale invariant. In shearing sheet simulations, the box size defines a scale; in a real disk, the disk scale height does. Furthermore, we point out that the mechanism analyzed by Waleffe (1997), whose qualitative and semi-quantitative relevance to the present work has been pointed out hereabove, is somewhat insensitive to the nature of the imposed boundary condition. Along with the results found in this paper, this suggests that only a scale rather than a specific boundary condition needs to be imposed for statistically stationary turbulence to show up in numerical simulations, as exemplified in Sect. 3.4. Finally, the role of an increasingly dominant Coriolis force is not to define another scale, which it cannot, but to modify the relative range of scales that are required for turbulence to exist (most likely because of its more and more stringent time-scale requirement), so that numerical resolution does play an important role in subcritical turbulence detection, as can be seen from Fig. 7 .

\subsection{Boundary conditions and aspect ratio}

Assessing the role of boundary conditions on the existence and properties of subcritical turbulence is an important question, since real accretion disk boundary conditions are not reproducible in experimental flows. However, the resolution demand in the local shearing box is already so large for a Keplerian flow that a global simulation of a subcritical Keplerian disk flow is totally out of reach. The best we can do is to compare numerical experiments with shearing sheet and rigid/periodic boundary conditions with one another, and with experimental results. This is the object of this section. 


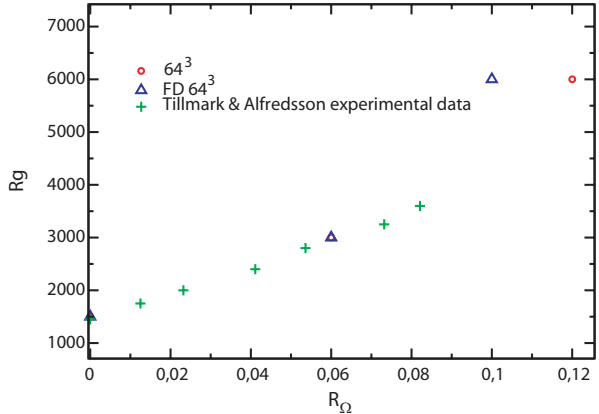

Fig. 14. $R g\left(R_{\Omega}\right)$ plot from experimental data (Tillmark \& Alfredsson 1996, crosses), and our numerical simulations using $64^{3}$ Fourier code (circles) and $64^{3}$ finite difference code (triangles) with cubic box and shearing sheet boundary conditions.

Before doing so, let us point out some important differences between the two types of boundary conditions:

- In the semi-Lagrangian variables defined by Eqs. (10), the only difference is that the velocity deviation from the laminar flow cancels on the rigid boundary in the rigid/periodic case, while it is periodic in the shear direction (as in the others) in the shearing sheet case. This results in a suppression of the boundary layer in the shearing sheet case.

- Characteristic sizes are the same in both cases. However, while for rigid/periodic conditions, structures are forced to remain more or less stationary with respect to the walls on average, this is not the case with shearing sheet boundary conditions, where structures can move at random through the boundary. As a consequence, a long-lasting mean flow distortion is apparent with rigid/periodic boundary conditions (due to the matching of turbulently enhanced transport with the viscous one in the boundary layer), while in shearing-sheet simulations, although such a distortion is usually locally found at any given time, it averages out over time, due to its random localization.

- This relates to a profound difference between accretion disks and actual experiments. In the latter, the flow profile adjusts to the imposed boundary condition through a pressure redistribution, and a stationary state is reached. In the former, this cannot take place, and the disk is never stationary, due to the resulting turbulent transport of mass and angular momentum.

In spite of these differences, we shall nevertheless argue that the choice of boundary conditions has only a limited impact on some of our qualitative and semi-quantitative results. This suggests that the underlying mechanisms are reasonably closely related in both settings, although much more work than what has been possible to do here is required to ascertain this conclusion.

\subsubsection{Cyclonic rotation}

Figure 14 displays a comparison of our numerical results with the Tillmark \& Alfredsson (1996) data, in the range of rotation number where these data were collected.

The agreement between the two is fair, with the Fourier code results being sensibly more compatible with the data than

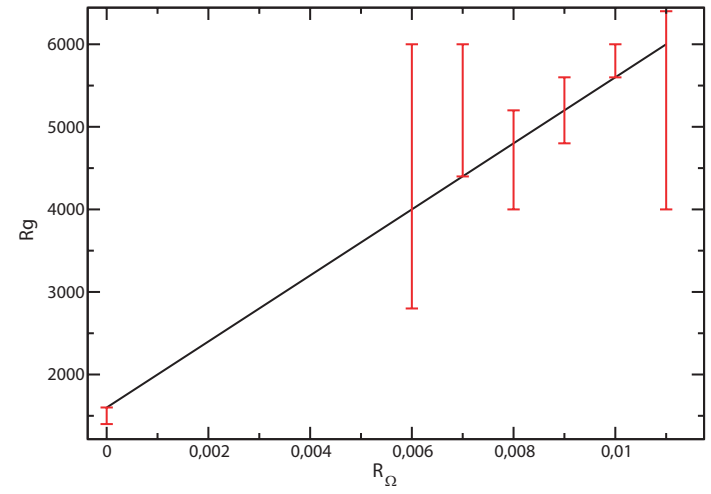

Fig. 15. $\mathrm{Re}_{\mathrm{c}}$ as a function of $R_{\Omega}$ for cyclonic rotating plane couette flow.

the finite difference code ones, at the larger rotation numbers. This follows because, at the same "resolution", a Fourier code is physically more resolved than a Finite difference code. Note also that some $128^{3}$ simulations were performed using the Fourier code and the same transition thresholds were found as for the $64^{3}$ simulations. This supports the idea that the $64^{3}$ Fourier code results are not resolution limited.

We have also made a few runs using rigid (shearwise direction) and periodic (other directions) boundary conditions with our ZEUS-like code. At each rotation number, we made a few tries with different Reynolds numbers to locate the transition threshold. Each run was computed from the same initial condition for 400 shear times with $80 \times 80 \times 40$ grid points and a $L_{x}=1.75 \pi L_{y}=1 L_{z}=1.2 \pi$ aspect ratio box (corresponding to the "minimal flow unit" aspect ratio, i.e. the smallest box in which turbulence can be sustained with these boundary conditions: see Hamilton et al. 1995, for details). The error bars upper bounds correspond to the lower Reynolds for which turbulence is found and the lower bound the higher Reynolds number for which turbulence is lost. The numerical data are shown in Fig. 15; the error bars reflect our poor sampling, not intrinsic fluctuations in the transition Reynolds number. These data are fitted by a linear law:

$R g=1400+4 \times 10^{5} R_{\Omega}$,

the slope of which is 15 times steeper than the one found from the experimental data.

This dramatic difference in transition Reynolds number with respect to the experimental and shearing sheet results is in fact controlled by the choice of the simulation box aspect ratio. For example, let us choose a longer box in the $z$ direction (i.e. $L_{x}=1.75 \pi L_{y}=1 L_{z}=2.4 \pi$ ). With such a choice, turbulence is sustained at $R_{\Omega}=0.01$ and $\operatorname{Re}=2400$, much closer to the expected transition Reynolds of Fig. 14 than what is predicted by Fig. 15. Finally, Komminaho et al. (1996), using a very elongated simulation box in the flow direction, found transition right at the experimentaly determined Reynolds number $\left(R g=3000, R_{\Omega}=0.06\right)$.

These result show the important role of aspect ratio in subcritical turbulence simulations with rigid/periodic boundary conditions. Apparently, the use of shearing sheet boundary conditions relaxes this constraint. This is reasonable since the 


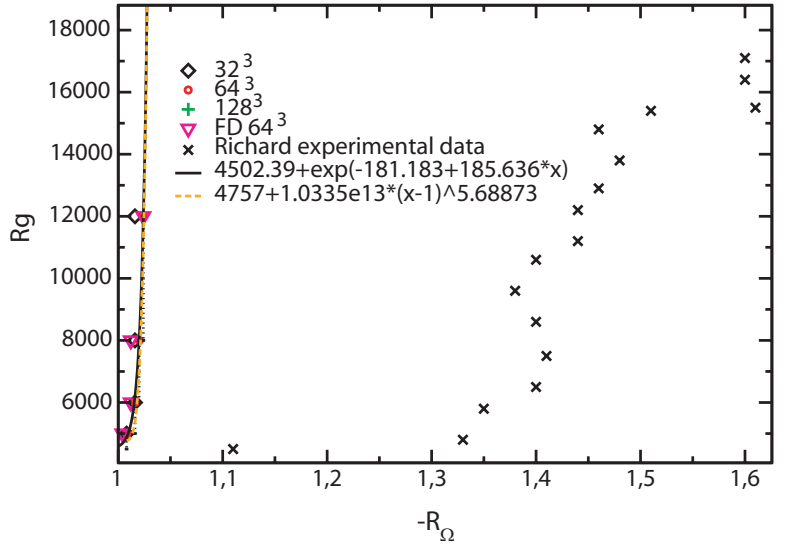

Fig. 16. $R g\left(R_{\Omega}\right)$ plot from experimental data on Taylor-Couette flows (Richard 2001, crosses), and the various numerical simulations results and related fits shown in Fig. 7.

shearing sheet box allows more freedom than rigid boundary conditions. In actual experiments, the aspect ratio is not an issue since usually very large $L_{x} / L_{y}$ and $L_{z} / l_{y}$ are used, so that the turbulence coherence length can freely adjust itself in these directions.

These results also indirectly suggest that the turbulence saturation mechanism is not strongly affected by the use of shearing sheet boundary conditions. One would nevertheless expect that the reduction of the shear in the middle of the flow, due to the mean velocity profile modification which occurs with rigid/periodic boundary conditions, produces a reduced turbulent transport. This is indeed the case: e.g., the turbulent transport at marginal stability $\left(R_{\Omega}=0\right)$ is $\left\langle v_{x} v_{y}\right\rangle \simeq 2 \times 10^{-3}(S d)^{2}$ for the rigid/periodic boundary conditions ${ }^{16}$, while one has $\left\langle v_{x} v_{y}\right\rangle \simeq 0.4(S d)^{2}$ throughout the flow with the shearing sheet boundary conditions, although the transition Reynolds number is the same in both instances. These features most probably find a natural explanation if the turbulence amplitude saturation mechanism is primarily controlled by the system nonlinearities, and not by the mean profile deformation.

\subsubsection{Anticyclonic rotation}

The comparison of our numerical results with Richard (2001) data is shown in Fig. 16.

The discrepancy between the experimental and numerical data is striking, especially at the light of the remarkable consistency observed for cyclonically rotating flows. In particular, the increase in transition Reynolds is considerably steeper in the numerical data than in the experimental ones. Note however that the numerical and experimental data seem to give the same transition Reynolds number at the marginal stability boundary.

Longaretti \& Dauchot (2005) have argued that the flow curvature plays no role in the anticyclonic flow data of Richard (2001), so that the origin of the large discrepancy between the

\footnotetext{
16 This is measured in the middle of the flow where the turbulent transport is maximized, and viscous transport negligible.
}

numerical and experimental results must be found elsewhere ${ }^{17}$. In this respect, note that experimental secondary flow distortions are much more likely to induce a linear instability somewhere in the flow on the anticyclonic side as on the cyclonic one. Indeed, recall that the stability limit is defined by Eq. (9). Consider the cyclonic marginal stability limit first $\left(R_{\Omega}=0\right)$, and assume that one moves away from it by imposing a small change in rotation $\delta \Omega$. The required change in shear profile $\delta S$ to locally achieve $2 \delta \Omega /(S(y)+\delta S)<0$ is large: $\delta S \sim S(y)$ is needed. Conversely, at the anticyclonic marginal stability limit $\left(R_{\Omega}=-1\right.$, i.e., $S=2 \Omega$ ), upon a small change $\delta \Omega$ of the rotation rate, a change $\delta S \simeq 2 \delta \Omega \ll S$ suffices to locally make $2 \Omega / S>-1$ and produce a linear instability somewhere in the system. This argument shows that the presence of secondary flows, such as Ekmann's circulation, can easily make the flow more unstable than it would be in its absence in anticyclonic flows, whereas this is much more difficult to achieve in cyclonic ones. This may easily explain the discrepancy between numerical and experimental results shown in Fig. 16, while the agreement is remarkable at the marginal stability boundary.

\section{Summary and conclusions}

The central results of this paper are displayed in Figs. 4, 5, 7-9, and their significance and implications are discussed in Sects. 3.3, 3.4, 4.1-4.4. The main implications of these results are summarized in the abstract. In the course of the discussion, we have found that a number incorrect statements have been made in the literature, most notably concerning the existence and importance of subcritical turbulence in presence of a dynamically significant Coriolis force. We have also found that resolution is a key issue for subcritical anticyclonically rotating flows (including Keplerian ones), and have quantified the relation between resolution, rotation and Reynolds number. In relation to this, we believe that the question of resolution of the dissipation scale is not emphasized enough in the astrophysics literature, and the potential effects of this problem are most probably underestimated.

Our simulations do not faithfully represent a real disk: neither vertical stratification, nor, more critically, realistic vertical boundary conditions have been implemented. A real (hydrodynamic disk) moves either in vacuum, or, more probably, in a hot corona. In both cases, one expects the real vertical boundary condition in the disk to be (nearly) stress-free. We have made some very preliminary simulations of ah stratified diskcorona system to test this idea, where most of the inertia lies in the disk. Although a strong numerical mixing of the corona and the disk at the interface prevents us to evolve the system for a long time $\left(50 t_{\mathrm{s}} \max \right)$, no significant difference in the overall dynamics of the disk did show up. However this problem probably requires more careful investigations to validate this conclusion.

Overall, the outcome of this investigation still leaves us with the issue of transport unresolved in MHD-inactive flows

\footnotetext{
17 In any case, the flow curvature always increases the transition Reynolds number, so that including curvature in the analysis of this problem can only make it worse, not better.
} 
(and possibly in some MHD-active ones), and we will briefly comment the various ways out of this conundrum.

We first note that an efficient enough local instability should lead to a large enough turbulent transport, because the transition to fully developed turbulence usually occurs to significantly lower Reynolds numbers in these systems than the ones found here. This is true, e.g., in rotating shear flows of the type considered here, in the linearly unstable regime. However, no such instability has yet been found in hydrodynamical Keplerian flows, either stratified or not, as discussed in the introduction. It remains to be seen whether another such instability can operate in hydrodynamic disks, but the list of potential driving agents has by now significantly been narrowed.

In what concerns the YSO disks dead-zone in particular, it may be that the disk stirring due to the MRI above and below the dead-zone itself (Fleming \& Stone 2003) might provide enough transport in the end if it excites large enough large scale 2D disturbances of the right type (Ioannou \& Kakouris 2001) in the disk. However, this option remains to be worked out in detail.

It has often been noted that transport in disks may not be due to turbulence but to waves (see, e.g., Papaloizou \& Lin 1995 for an introduction to the subject). Recent results on the existence of vortices in stratified disks (Barranco \& Marcus 2005 ) and on the coupling of waves to vortices resulting in efficient transport in 2D dynamics (Bodo et al. 2005 and references therein) support this idea.

\section{Appendix A: Displaced particle analysis for rotating flows}

The following line of argument closely follows Tritton \& Davies (1981) and Tritton (1992). Let us consider a rotating shear flow, whose dynamics is controlled by Eq. (1). As in Sect. 2.1, $x$ is the direction of the flow, $y$ the direction of the shear, and $z$ the direction perpendicular to the $x, y$ plane, in which the rotation $\mathbf{\Omega}$ is applied. The laminar equilibrium velocity $\boldsymbol{u}_{L}=(U(y), 0,0)$ generates a Coriolis force in the $y$ direction of magnitude $-2 \rho \Omega U$ (in algebraic value), which is balanced by the equilibrium generalized pressure gradient $-\mathrm{d} \pi / \mathrm{d} y$.

Let us further consider two fluid "rods" of infinite extent in the streamwise direction $x$, and located at positions $y_{1}$ and $y_{2}=y_{1}+\delta y$. The streamwise velocities of these rods are $U_{1}$ and $U_{2}$, respectively. Let us assume that one displaces the rod at $y_{1}$ to location $y_{2}$, without disturbing the pressure distribution. Although the total work of the Coriolis force vanishes, there is a net partial work due to the force component in the $x$ direction which originates in the velocity $v$ of this displacement in the $y$ direction. Because of this partial work, the rod experiences a change of $x$ momentum, and therefore of $x$ velocity, which reads

$U_{1}^{\prime}-U_{1}=\int 2 \Omega v \mathrm{~d} t=2 \Omega \delta y$,

so that the velocity $U_{1}^{\prime}$ of the rod when it reaches location $y_{2}$ differs from the equilibrium velocity $U_{2}$, and correlatively, the $x$ component of the Coriolis force acting on this displaced

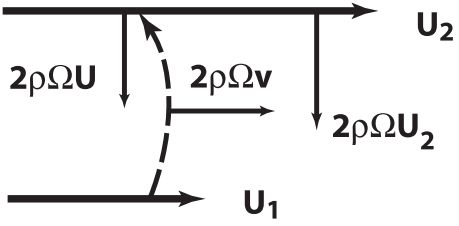

Fig. A.1. Sketch of the effect of the Coriolis force on the displaced particle (see text).

rod, $-2 \rho \Omega U_{1}^{\prime}$ (in algebraic value) differs from the equilibrium one, $-2 \rho \Omega U_{2}$ (see Fig. A.1).

Consequently, the net result between the equilibrium pressure gradient and the Coriolis force will tend to restore the displaced rod to its equilibrium position ${ }^{18}$ if $U_{1}^{\prime}>U_{2}$, or displace it further if $U_{1}^{\prime}<U_{2}$. From Eqs. (A.1) and (3), one obtains

$U_{1}^{\prime}-U_{2}=2 \Omega \delta y-\frac{\mathrm{d} U}{\mathrm{~d} y} \delta y=S\left(R_{\Omega}+1\right) \delta y$,

where $S=-\mathrm{d} U / \mathrm{d} y$ is the shear. From this result, the net force (Coriolis and pressure) on the displaced rod reads

$2 \rho \Omega\left(U_{2}-U_{1}^{\prime}\right)=-\rho S^{2} R_{\Omega}\left(R_{\Omega}+1\right) \delta y$.

This shows that equilibrium is always restored when $R_{\Omega}>0$ or $R_{\Omega}<-1$ and destroyed otherwise (equality holds at marginal stability). This is the result quoted in Sect. 2.1. This result can also be directly derived from the linearized eulerian equation of motion with the use of spatially uniform perturbations of the pressure and the velocity.

Acknowledgements. The undertaking and completion of this work has benefitted from discussions with a number of colleagues in the past few years, most notably O. Dauchot, F. Daviaud, B. Dubrulle, F. Lignières, and J.-P. Zahn. PYL also acknowledges discussions held at the KITP conference on the physics of astrophysical outflows and accretion disks, with C. Gammie, J. Hawley, B. Johnson, E. Quataert, and J. Goodman. Interactions with O. Blaes and G. Bodo have been particularly fruitful. David Clarke has provided us with his version of the ZEUS code, and his friendly help (as well as G. Bodo's) in the initial phases of this project is gratefully acknowledged. We thank Françoise Roch, Françoise Berthoud and Alain Pasturel for their help in accessing the computing resources of the SCCI of the Grenoble Observatory, and of the PHYNUM and MEDETPHY platforms of the Grenoble University CIMENT project. A large fraction of the simulations presented here has also been performed at IDRIS (French national computational center).

\section{References}

Afshordi, N., Mukhopadhyay, B., \& Narayan, R. 2005, ApJ, 629, 373 Arlt, R., \& Urpin, V. 2004, A\&A, 426, 755

Balbus, S. A. 2003, ARA\&A, 41, 555

Balbus, S. A. 2004, A\&A, submitted [arXiv: astro-ph/0408510]

Balbus, S. A., \& Hawley, J. F. 1991, ApJ, 376, 214

Balbus, S. A., Hawley, J. F., \& Stone, J. M. 1996, ApJ, 467, 76

Barranco, J. A., \& Marcus, P. S. 2005, ApJ, 623, 1157

${ }^{18}$ Consider the direction and magnitude of the Coriolis force and pressure force along the $y$ axis to derive the effect of the inequality. 
Bech, K. H., \& Andersson, H. I. 1997, J. Fluid Mech., 347, 289

Blaes, O. M. 1987, MNRAS, 227, 975

Bodo, G., Chagelishvili, G., Murante, G., et al. 2005, A\&A, 437, 9

Bottin, S., Dauchot, O., \& Daviaud, F. 1997, Phys. Rev. Lett., 79, 4377

Brandenburg, A., \& Dintrans, B. 2001 [arXiv:astro-ph/0111313]

Brosa, U., \& Grossmann, S. 1999, European Phys. J. B, 9, 343

Cabot, W. 1996, ApJ, 465, 874

Cambon, C., Benoit, J., Shao, L., \& Jacquin, L. 1994, J. Fluid Mech., 278,175

Chagelishvili, G. D., Zahn, J.-P., Tevzadze, A. G., \& Lominadze, J. G. 2003, A\&A, 402, 401

Darbyshire, A. G., \& Mullin, T. 1995, J. Fluid Mech., 289, 83

Dauchot, O., \& Daviaud, F. 1995a, Phys. Fluids, 7, 335

Dauchot, O., \& Daviaud, F. 1995b, Phys. Fluids, 7, 901

Daviaud, F., Hegseth, J., \& Bergé, P. 1992, Phys. Rev. Lett., 69, 2511

Drazin, P., \& Reid, W. 1981, Hydrodynamic stability (Cambridge Univ. Press)

Dubrulle, B. 1993, Icarus, 106, 59

Dubrulle, B., \& Zahn, J.-P. 1991, J. Fluid Mech., 231, 561

Dubrulle, B., Dauchot, O., Daviaud, F., et al. 2005a, Phys. Fluids, accepted

Dubrulle, B., Marié, L., Normand, C., et al. 2005b, A\&A, 429, 1

Eckhardt, B., \& Mersmann, A. 1999, Phys. Rev. E, 60, 509

Faisst, H., \& Eckhardt, B. 2004, J. Fluid Mech., 504, 343

Fleming, T., \& Stone, J. M. 2003, ApJ, 585, 908

Gammie, C. F. 1996, ApJ, 457, 355

Garaud, P., \& Ogilvie, G. I. 2005, J. Fluid Mech., submitted [arXiv: astro-ph/0503223]

Goldreich, P., \& Schubert, G. 1967, ApJ, 150, 571

Goodman, J., \& Balbus, S. A. 2001 [arXiv: astro-ph/0110229]

Grossman, S. 2000, Rev. Mod. Phys., 72, 603

Hamilton, J. M., Kim, J., \& Waleffe, F. 1995, J. Fluid Mech., 287, 317

Hawley, J. F. 1991, ApJ, 381, 496

Hawley, J. F., Balbus, S. A., \& Winters, W. F. 1999, ApJ, 518, 394

Hawley, J. F., Gammie, C. F., \& Balbus, S. A. 1995, ApJ, 440, 742

Hersant, F., Dubrulle, B., \& Huré, J.-M. 2005, A\&A, 429, 531

Ioannou, P. J., \& Kakouris, A. 2001, ApJ, 550, 931

Johnson, B. M., \& Gammie, C. F. 2005a, ApJ, 626, 978

Johnson, B. M., \& Gammie, C. F. 2005b, in preparation

Klahr, H. 2004, ApJ, 606, 1070

Klahr, H. H., \& Bodenheimer, P. 2003, ApJ, 582, 869

Komminaho, J., Lundbladh, A., \& Johansson, A. V. 1996, J. Fluid Mech., 320, 259

Leblanc, S., \& Cambon, C. 1997, Phys. Fluids, 9, 1307

Lerner, J., \& Knobloch, E. 1988, J. Fluid Mech., 189, 117

Lesieur, M. 1990, Turbulence in fluids third edition (Kluwer)

Longaretti, P.-Y. 2002, ApJ, 576, 587
Longaretti, P.-Y., \& Dauchot, O. 2005, in Proc. Bristol 2004 Symposium on the laminar-turbulent transition, ed. Kerswell \& Mullin (Kluwer)

Mukhopadhyay, B., Afshordi, N., \& Narayan, R. 2005, ApJ, 629, 383

Ogilvie, G. I. 2003, MNRAS, 340, 969

Papaloizou, J. C. B., \& Lin, D. N. C. 1995, ARA\&A, 33, 505

Papaloizou, J. C. B., \& Pringle, J. E. 1984, MNRAS, 208, 721

Pedley, T. J. 1969, J. Fluid Mech., 35, 97

Peyret, R. 2002, Spectral Methods for Incompressible Viscous Flow (Springer)

Pumir, A. 1996, Phys. Fluids, 8, 3112

Rüdiger, G., Arlt, R., \& Shalybkov, D. 2002, A\&A, 391, 781

Richard, D. 2001, Ph.D. Thesis, Université de Paris VII

Richard, D., \& Zahn, J. 1999, A\&A, 347, 734

Richard, D., Dauchot, O., \& Zahn, J.-P. 2001, in Proc. of the 12th Couette-Taylor Workshop, Evanston, USA

Rogallo, R. S. 1981, NASA STI/Recon Technical Report N, 81, 31508

Romanov, V. A. 1973, Func. Anal. Appl., 7, 137

Salhi, A., \& Cambon, C. 1997, J. Fluid Mech., 347, 171

Satomura, T. 1981, J. Meteor. Soc. Japan, 59, 148

Schmiegel, A., \& Eckhardt, B. 1997, Phys. Rev. Lett., 79, 5250

Shakura, N. I., Sunyaev, R. A., \& Zilitinkevich, S. S. 1978, A\&A, 62, 179

Shalybkov, D., \& Ruediger, G. 2005, A\&A, 438, 411

Sipp, D., \& Jacquin, L. 2000, Phys. Fluids, 12, 1740

Speziale, C. G. 1991, Ann. Rev. Fluid Mech., 23, 107

Speziale, C. G., \& Mhuiris, N. M. 1989, Phys. Fluids, 1, 294

Stone, J. M., \& Balbus, S. A. 1996, ApJ, 464, 364

Stone, J. M., \& Norman, M. L. 1992, ApJS, 80, 753

Taylor, G. I. 1936, Proc. Roy. Soc. London A, 157, 546

Tevzadze, A. G., Chagelishvili, G. D., Zahn, J.-P., Chanishvili, R. G., \& Lominadze, J. G. 2003, A\&A, 407, 779

Tillmark, N., \& Alfredsson, P. H. 1996, in Advances in Turbulence VI., ed. S. Gavrilakis, L. Machiels, \& P. A. Monkewitz (Kluwer), 391

Tritton, D. J. 1992, J. Fluid Mech., 241, 503

Tritton, D. J., \& Davies, P. A. 1981, in Hydrodynamic instabilities and the transition to turbulence (Springer-Verlag), 229

Umurhan, O. M. 2005, MNRAS, submitted [arXiv: astro-ph/0506016]

Umurhan, O. M., \& Regev, O. 2004, A\&A, 427, 855

Urpin, V. 2003, A\&A, 404, 397

Waleffe, F. 1995, Phys. Fluids, 7, 3060

Waleffe, F. 1997, Phys. Fluids, 9, 883

Waleffe, F. 2003, Phys. Fluids, 15, 1517

Wendt, G. 1933, Ing. Arch., 4, 577

Yecko, P. A. 2004, A\&A, 425, 385 